\title{
LONG TIME BEHAVIOR OF A TWO-PHASE OPTIMAL DESIGN FOR THE HEAT EQUATION
}

\author{
GRÉGOIRE ALLAIRE*, ARNAUD MÜNCH ${ }^{\dagger}$, AND FRANCISCO PERIAGO
}

\begin{abstract}
We consider a two-phase isotropic optimal design problem within the context of the transient heat equation. The objective is to minimize the average of the dissipated thermal energy during a fixed time interval $[0, T]$. The time-independent material properties are taken as design variables. A full relaxation for this problem was established in [Relaxation of an optimal design problem for the heat equation, JMPA 89 (2008)] by using the homogenization method. In this paper, we study the asymptotic behavior as $T$ goes to infinity of the solutions of the relaxed problem and prove that they converge to an optimal relaxed design of the corresponding two-phase optimization problem for the stationary heat equation. Next, we study necessary optimality conditions for the relaxed optimization problem under the transient heat equation and use those to characterize the micro-structure of the optimal designs, which appears in the form of a sequential laminate of rank at most $N$, the spatial dimension. An asymptotic analysis of the optimality conditions let us prove that, for $T$ large enough, the order of lamination is in fact of at most $N-1$. Several numerical experiments in $2 D$ complete our study.
\end{abstract}

Key words. Optimal design, Heat equation, Relaxation by the homogenization method, Asymptotic behavior, Optimality conditions, Numerical simulation

AMS subject classifications. 35B40, 35K05, 49J45, 65M60, 74Q10

1. Introduction and problem statement. Optimal design problems in which the goal is to know the best way of mixing two different materials in order to optimize some physical quantity associated with the resulting structure have been extensively studied during the last decades, mainly in the case where the underlying state equation is elliptic $[6,13]$. A common feature of these optimal design problems is that they usually are ill-posed in the sense that minimizing sequences for the objective function exhibit finer and finer micro-structure. Among the techniques and tools used to deal with this type of problems, homogenization and variational formulations have played an important role (see also $[1,3,15,18]$ ). More recently, optimal design problems for time-dependent designs and time-dependent state equations - mainly of hyperbolic type - have been also considered $([5,8,9,10])$. In particular, in [8] a class of spatialtemporal composite materials (rank-1 and rank-2 spatial-temporal laminates) were introduced. See also [9] for some physical examples.

This paper is concerned with the heat equation, as a continuation of [11] in some specific directions where the following problem, parameterized by the final time $T>0$, is addressed:

$$
\left(\mathrm{P}_{T}\right) \quad \text { Minimize in } \mathcal{X} \in \mathbf{C D}: \quad J_{T}(\mathcal{X})=\frac{1}{T} \int_{0}^{T} \int_{\Omega} K(x) \nabla u(t, x) \cdot \nabla u(t, x) d x d t
$$

${ }^{*}$ Centre de Mathématiques Appliquées. École Polytechnique. 91128 Palaiseau, France (gregoire.allaire@polytechnique.fr). Member of the DEFI project at INRIA Saclay Ile-deFrance, partially supported by the Chair "Mathematical modelling and numerical simulation, FEADS - École Polytechnique - INRIA".

${ }^{\dagger}$ Laboratoire de Mathématiques de Clermont-Ferrand, UMR CNRS 6620, Université Blaise Pascal, Campus des Cézeaux, 63177 Aubière cedex, France (arnaud.munch@math.univ-bpclermont.fr). Partially supported by grants ANR-07-JC-0139-01 (Agence national de la recherche, France) and 08720/PI/08 from Fundacion Séneca (Gobernio regional de Murcia, Spain).

${ }^{\ddagger}$ Departamento de Matemática Aplicada y Estadística, ETSI Industriales, Universidad Politécnica de Cartagena, 30203 Cartagena, Spain - f .periago@upct.es. Supported by projects MTM2007-62945 from Ministerio de Educación y Ciencia (Spain) and 08720/PI/08 from Fundación Séneca (Agencia de Ciencia y Tecnología de la Región de Murcia (Spain). II PCTRM 2007-10). 
where the state variable $u=u(t, x)$ is the solution of the system

$$
\left\{\begin{array}{lll}
\beta(x) u^{\prime}(t, x)-\operatorname{div}(K(x) \nabla u(t, x))=f(t, x) & \text { in } \quad(0, T) \times \Omega \\
u=0 & \text { on } \quad(0, T) \times \partial \Omega \\
u(0, x)=u_{0}(x) & \text { in } \quad \Omega,
\end{array}\right.
$$

where $u^{\prime}$ denotes the partial time derivative of $u$, and

$$
\left\{\begin{array}{l}
\beta(x)=\mathcal{X}(x) \beta_{1}+(1-\mathcal{X}(x)) \beta_{2} \\
K(x)=\mathcal{X}(x) k_{1} I_{N}+(1-\mathcal{X}(x)) k_{2} I_{N}
\end{array}\right.
$$

The design variable $\mathcal{X}$ is the characteristic function of the region occupied by the material $\left(\beta_{1}, k_{1}\right)$. It belongs to the class of classical designs $\mathbf{C D}$ defined as

$$
\mathbf{C D}=\left\{\mathcal{X} \in L^{\infty}(\Omega ;\{0,1\}) \text { s.t. } \int_{\Omega} \mathcal{X}(x) d x=L|\Omega|\right\}
$$

for some fixed $0<L<1$. The domain $\Omega \subset \mathbb{R}^{N}$ is assumed to be smooth and bounded. The two phases are homogeneous, isotropic and with mass densities $\rho_{i}>0$, specific heats $c_{i}>0$, and thermal conductivities $k_{i}>0, i=1,2$. Without loss of generality we assume $k_{1}<k_{2}$. We then define $\beta_{i}=\rho_{i} c_{i}, i=1,2$. $I_{N}$ denotes the identity matrix of order $N, f$ is the heat source, $u_{0}$ the initial temperature, and $u(t, x)$ the temperature at position $x$ and time $t$. For $f \in L^{2}((0, T) \times \Omega)$ and $u_{0} \in L^{2}(\Omega)$, system (1.1) is well-posed in the class $u \in C\left([0, T] ; L^{2}(\Omega)\right) \cap L^{2}\left((0, T) ; H_{0}^{1}(\Omega)\right)$. The cost $J_{T}$ represents an average of the dissipated thermal energy during the time interval $[0, T]$. We refer to $[19,20]$ where a similar problem with a functional cost depending on $u$ is addressed under an engineering viewpoint.

As indicated above, problem $\left(\mathrm{P}_{T}\right)$ is usually ill-posed in the sense that there are no minimizers in the space of classical designs CD (see [12] for some related problems). Using homogenization theory, in [11, Th. 2.4], the following relaxed formulation for $\left(\mathrm{P}_{T}\right)$ was found:

$$
\left(\mathrm{RP}_{T}\right) \quad \text { Minimize in }\left(\theta, K^{\star}\right) \in \mathbf{R D}: \quad J_{T}^{\star}\left(\theta, K^{\star}\right)
$$

where RD designates the space of relaxed designs (detailed in Section 2).

The main goal of this work is to analyze, both theoretically and numerically, the behavior of the optimal relaxed designs $\left(\theta, K^{\star}\right)=\left(\theta_{T}, K_{T}^{\star}\right)$ as the variable $T$ goes to infinity. Assuming that the heat source $f(x)$ does not depend on time, the unique solution of (1.1) converges as $t \rightarrow+\infty$ to $\bar{u} \in H_{0}^{1}(\Omega)$, solution of the stationary equation

$$
\left\{\begin{array}{lll}
-\operatorname{div}(K(x) \nabla \bar{u}(x))=f(x) & \text { in } & \Omega \\
u=0 & \text { on } & \partial \Omega .
\end{array}\right.
$$

Associated with this PDE we consider the design problem

$$
\left(\mathrm{P}_{\infty}\right) \quad \text { Minimize in } \mathcal{X} \in \mathbf{C D}: \quad J_{\infty}(\mathcal{X})=\int_{\Omega} K(x) \nabla \bar{u}(x) \cdot \nabla \bar{u}(x) d x .
$$

A relaxed formulation $\left(R P_{\infty}\right)$ of $\left(P_{\infty}\right)$ is by now very well-understood (see [1] and next section). In particular, an optimal micro-structure can always be found among first order laminates. 
The outline of the paper is as follows. In Section 2, we first briefly remind the formulations $\left(R P_{T}\right),\left(R P_{\infty}\right)$ and introduce some notation. In Section 3, we prove that any weak limit (as $T$ goes to infinity) of a converging subsequence of $\left(\theta_{T}, K_{T}^{\star}\right)_{T>0}$ is in fact an optimal design of $\left(R P_{\infty}\right)$. In Section 4 , we study a necessary optimality condition for the parabolic problem $\left(R P_{T}\right)$ and prove that optimal micro-structures for this problem can be found among laminates of at most rank $N$, the spatial dimension. Section 5 contains an asymptotic analysis when $T \rightarrow \infty$ of that necessary optimality condition. As a result, we obtain that, for $T$ large enough, the order of lamination of optimal designs for $\left(R P_{T}\right)$ is at most $N-1$. Numerical experiments are given in Section 6 . We deduce from this analysis that the homogenization process and the limit as $T$ goes to infinity commute (see Figure 1.1).

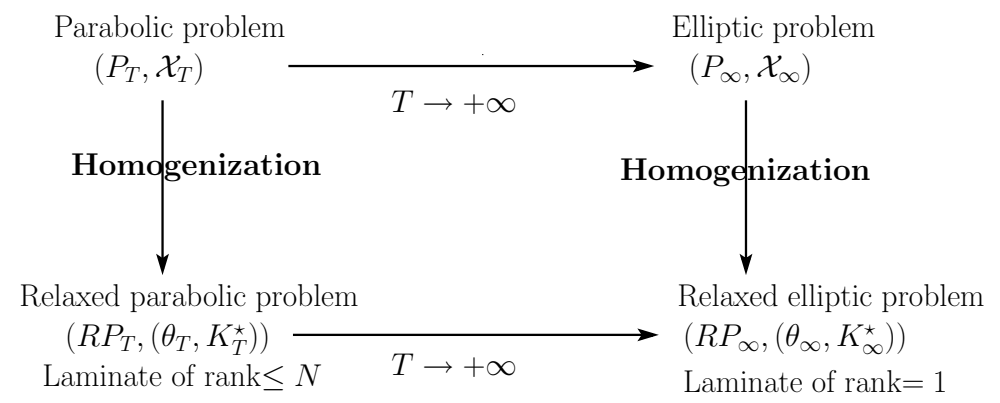

Fig. 1.1. Commutation between Homogenization process and limit of the heat system as $T \rightarrow \infty$.

2. Relaxed formulations for $\left(P_{T}\right)$ and $\left(P_{\infty}\right)$. We recall here briefly the relaxed formulations, derived from homogenization theory, associated with $\left(P_{T}\right)$ and $\left(P_{\infty}\right)$. To this end, we firstly introduce the space of relaxed designs

$$
\mathbf{R D}=\left\{\left(\theta, K^{\star}\right) \in L^{\infty}\left(\Omega ;[0,1] \times \mathcal{M}_{N}^{s}\right): K^{\star}(x) \in G_{\theta(x)} \text { a.e. } x \in \Omega,\|\theta\|_{L^{1}}=L|\Omega|\right\},
$$

where $\mathcal{M}_{N}^{s}=\mathcal{M}_{N}^{s}\left(k_{1}, k_{2}\right)$ is the space of real symmetric matrices $M$ of order $N$ satisfying, for all $\xi \in \mathbb{R}^{N}, k_{1}|\xi|^{2} \leq M \xi \cdot \xi \leq k_{2}|\xi|^{2}$. For a given $\theta \in L^{\infty}(\Omega ;[0,1])$, the so-called $G_{\theta}$-closure is the set of all symmetric matrices with eigenvalues $\lambda_{1}, \cdots, \lambda_{N}$ satisfying

$$
\left\{\begin{array}{l}
\lambda_{\theta}^{-} \leq \lambda_{j} \leq \lambda_{\theta}^{+}, \quad 1 \leq j \leq N \\
\sum_{j=1}^{N} \frac{1}{\lambda_{j}-k_{1}} \leq h_{\theta}^{-}=\frac{1}{\lambda_{\theta}^{-}-k_{1}}+\frac{N-1}{\lambda_{\theta}^{+}-k_{1}} \\
\sum_{j=1}^{N} \frac{1}{k_{2}-\lambda_{j}} \leq h_{\theta}^{+}=\frac{1}{k_{2}-\lambda_{\theta}^{-}}+\frac{N-1}{k_{2}-\lambda_{\theta}^{+}}
\end{array}\right.
$$

where $\lambda_{\theta}^{-}=\left(\frac{\theta}{k_{1}}+\frac{1-\theta}{k_{2}}\right)^{-1}$ is the harmonic mean and $\lambda_{\theta}^{+}=\theta k_{1}+(1-\theta) k_{2}$ the arithmetic mean of $\left(k_{1}, k_{2}\right)$. For more details we refer to [1].

Second, we consider the relaxed cost

$$
J_{T}^{\star}\left(\theta, K^{\star}\right)=\frac{1}{T} \int_{0}^{T} \int_{\Omega} K^{\star}(x) \nabla u(t, x) \cdot \nabla u(t, x) d x d t,
$$


where now $u$ is the solution of

$$
\left\{\begin{array}{lll}
\beta^{\star}(x) u^{\prime}(t, x)-\operatorname{div}\left(K^{\star}(x) \nabla u(t, x)\right)=f(t, x) & \text { in }(0, T) \times \Omega \\
u=0 & \text { on }(0, T) \times \partial \Omega \\
u(0, x)=u_{0}(x) & \text { in } \Omega,
\end{array}\right.
$$

with $\beta^{\star}(x)=\theta(x) \beta_{1}+(1-\theta(x)) \beta_{2}$. Homogenization theory provides the following two results (see [11, Th. 2.4] and [1, Th. 3.2.1 and Th. 3.2.6], respectively).

Theorem 2.1 (Parabolic case for $T$ fixed). Consider the following problem

$$
\left(R P_{T}\right) \text { Minimize in }\left(\theta, K^{\star}\right) \in \mathbf{R D}: \quad J_{T}^{\star}\left(\theta, K^{\star}\right) .
$$

$\left(R P_{T}\right)$ is a relaxation of $\left(P_{T}\right)$ in the following sense:

(i) there exists at least one minimizer for $\left(R P_{T}\right)$ in the space $\mathbf{R D}$,

(ii) up to a subsequence, every minimizing sequence of classical designs $\mathcal{X}_{n}$ converges, weak- $\star$ in $L^{\infty}(\Omega ;[0,1])$, to a relaxed density $\theta$, and its associated sequence of tensors

$$
K_{n}=\mathcal{X}_{n} k_{1} I_{N}+\left(1-\mathcal{X}_{n}\right) k_{2} I_{N}
$$

$H$-converges to an effective tensor $K^{\star}$ such that $\left(\theta, K^{\star}\right)$ is a minimizer for $\left(R P_{T}\right)$, and

(iii) conversely, every relaxed minimizer $\left(\theta, K^{\star}\right) \in \mathbf{R D}$ of $\left(R P_{T}\right)$ is attained by a minimizing sequence $\mathcal{X}_{n}$ of $\left(P_{T}\right)$ in the sense that

$$
\left\{\begin{array}{l}
\mathcal{X}_{n} \rightarrow \theta \quad \text { weak } \star \text { in } L^{\infty}(\Omega), \\
K_{n} \stackrel{H}{\rightarrow} K^{\star} .
\end{array}\right.
$$

Here $\stackrel{\mathrm{H}}{\rightarrow}$ stands for the convergence in the sense of homogenization (see $[1$, Definition 1.2.15]). Note that a minimizer of $\left(\mathrm{RP}_{T}\right)$ depends on the final time $T$. To make clearer this dependance, from now on, we shall denote such a minimizer by $\left(\theta_{T}, K_{T}^{\star}\right)$.

Theorem 2.2 (Elliptic case). Consider the following problem

$$
\left(R P_{\infty}\right) \quad \text { Minimize in }\left(\theta, K^{\star}\right) \in \mathbf{R D}: \quad J_{\infty}^{\star}\left(\theta, K^{\star}\right)=\int_{\Omega} K^{\star}(x) \nabla \bar{u}(x) \cdot \nabla \bar{u}(x) d x
$$

where $\bar{u} \in H_{0}^{1}(\Omega)$ solves

$$
\left\{\begin{array}{lll}
-\operatorname{div}\left(K^{\star} \nabla \bar{u}\right)=f & \text { in } & \Omega \\
\bar{u}=0 & \text { on } & \partial \Omega .
\end{array}\right.
$$

$(R P)_{\infty}$ is a relaxation of $\left(P_{\infty}\right)$ is the sense of the previous theorem. Moreover, an optimal effective tensor for $\left(R P_{\infty}\right)$ can be obtained as a first-order laminate in any direction orthogonal to $\nabla \bar{u}$.

3. Asymptotics for $T \rightarrow \infty$. We assume henceforth that $f(x) \in L^{2}(\Omega)$ is time independent. Let $\left\{T_{n}\right\}_{n \in \mathbb{N}}$ be an increasing sequence of positive times converging to infinity. For each $T_{n}$, problem $\left(\mathrm{RP}_{T_{n}}\right)$ has (at least) a minimizer $\left(\theta_{T_{n}}, K_{T_{n}}^{\star}\right) \in \mathbf{R D}$. Since $\left(\theta_{T_{n}}, K_{T_{n}}^{\star}\right)$ is bounded in $L^{\infty}\left(\Omega ;[0,1] \times \mathcal{M}_{N}^{s}\left(k_{1}, k_{2}\right)\right)$, up to a subsequence still labeled by $n$, we have, as $n \rightarrow+\infty$,

$$
\left\{\begin{array}{lll}
\theta_{T_{n}} & \rightarrow & \theta_{T_{\infty}} \text { weak- } \star \text { in } L^{\infty}(\Omega ;[0,1]), \\
K_{T_{n}}^{\star} & \stackrel{\mathrm{H}}{\rightarrow} & K_{T_{\infty}}^{\star}
\end{array}\right.
$$


Our task in this section is to prove that $\left(\theta_{T_{\infty}}, K_{T_{\infty}}^{\star}\right)$ is an optimal solution of $\left(\mathrm{RP}_{\infty}\right)$. We shall need the following preliminary result.

Lemma 3.1. Let $u_{n}$ be the solution of

$$
\left\{\begin{array}{lll}
\beta_{n}^{\star}(x) u_{n}^{\prime}(t, x)-\operatorname{div}\left(K_{T_{n}}^{\star}(x) \nabla u_{n}(t, x)\right)=f(x) & \text { in }(0, \infty) \times \Omega \\
u_{n}=0 & \text { on }(0, \infty) \times \partial \Omega \\
u_{n}(0, x)=u_{0}(x) & \text { in } \Omega,
\end{array}\right.
$$

with $\beta_{n}^{\star}(x)=\theta_{T_{n}}(x) \beta_{1}+\left(1-\theta_{T_{n}}(x)\right) \beta_{2}$. Then,

$$
\lim _{n \rightarrow \infty} \frac{1}{T_{n}} \int_{0}^{T_{n}} \int_{\Omega} K_{T_{n}}^{\star} \nabla u_{n} \cdot \nabla u_{n} d x d t=\int_{\Omega} K_{T_{\infty}}^{\star} \nabla \bar{u}_{\infty} \cdot \nabla \bar{u}_{\infty} d x
$$

where $\bar{u}_{\infty}(x) \in H_{0}^{1}(\Omega)$ is the solution of

$$
\left\{\begin{array}{lll}
-\operatorname{div}\left(K_{T_{\infty}}^{\star}(x) \nabla \bar{u}_{\infty}(x)\right)=f(x) & \text { in } & \Omega \\
\bar{u}_{\infty}=0 & \text { on } & \partial \Omega
\end{array}\right.
$$

Proof. We begin by proving that there exist two constants $C_{1}, C_{2}>0$, independent of $n$, such that

$$
\left\|u_{n}(t)-\bar{u}_{n}\right\|_{L^{2}(\Omega)} \leq C_{1} e^{-C_{2} t}, \quad t>0
$$

where $\bar{u}_{n}$ solves

$$
\left\{\begin{array}{lll}
-\operatorname{div}\left(K_{T_{n}}^{\star} \nabla \bar{u}_{n}\right)=f & \text { in } & \Omega \\
\bar{u}_{n}=0 & \text { on } & \partial \Omega .
\end{array}\right.
$$

The function $v_{n}(t, x)=u_{n}(t, x)-\bar{u}_{n}(x)$ solves

$$
\left\{\begin{array}{lll}
\beta_{n}^{\star}(x) v_{n}^{\prime}(t, x)-\operatorname{div}\left(K_{T_{n}}^{\star}(x) \nabla v_{n}(t, x)\right)=0 & \text { in } & (0, \infty) \times \Omega \\
v_{n}=0 & \text { on } & (0, \infty) \times \partial \Omega \\
v_{n}(0, x)=u_{0}(x)-\bar{u}_{n}(x) & \text { in } & \Omega .
\end{array}\right.
$$

Using the spectral decomposition,

$$
v_{n}(t, x)=\sum_{k=1}^{\infty} a_{n}^{k} e^{-\lambda_{n}^{k} t} \omega_{n}^{k}(x)
$$

where $\omega_{n}^{k} \in H_{0}^{1}(\Omega)$, with $\left\|\omega_{n}^{k}\right\|_{L_{\beta_{n}^{\star}}^{2}(\Omega)}^{2}=\int_{\Omega} \beta_{n}^{\star}\left|\omega_{n}^{k}\right|^{2} d x=1$, are the normalized eigenfunctions of the boundary-value problem

$$
\begin{cases}-\operatorname{div}\left(K_{T_{n}}^{\star} \nabla \omega_{n}^{k}\right)=\lambda_{n}^{k} \beta_{n}^{\star} \omega_{n}^{k} & \text { in } \quad \Omega \\ \omega_{n}^{k}=0 & \text { on } \quad \partial \Omega,\end{cases}
$$

with $0<\lambda_{n}^{1}<\lambda_{n}^{2} \leq \lambda_{n}^{3} \leq \cdots$, its associated eigenvalues, and

$$
a_{n}^{k}=\int_{\Omega} \beta_{n}^{\star}(x)\left(u_{0}(x)-\bar{u}_{n}(x)\right) \omega_{n}^{k}(x) d x, \quad k, n \in \mathbb{N} .
$$


Using that $\beta_{1} \leq \beta_{n}^{\star}(x)$ a.e. $x \in \Omega$ and Parseval's identity, we have

$$
\begin{aligned}
\beta_{1}\left\|v_{n}(t)\right\|_{L^{2}(\Omega)}^{2} & \leq\left\|v_{n}(t)\right\|_{L_{\beta_{n}^{\star}}^{2}(\Omega)}^{2} \\
& =\sum_{k=1}^{\infty} e^{-2 \lambda_{n}^{k} t}\left|a_{n}^{k}\right|^{2} \\
& \leq e^{-2 \lambda_{n}^{1} t}\left\|u_{0}-\bar{u}_{n}\right\|_{L_{\beta_{n}^{\star}}^{2}(\Omega)}^{2} .
\end{aligned}
$$

Since $K_{T_{n}}^{\star} \stackrel{\mathrm{H}}{\rightarrow} K_{T_{\infty}}^{\star}$ and $0<\beta_{1} \leq \beta_{n}^{\star}(x) \leq \beta_{2}$ a.e. $x \in \Omega$, the term $\left\|u_{0}-\bar{u}_{n}\right\|_{L_{\beta_{n}^{\star}}^{2}(\Omega)}^{2}$ is uniformly bounded. Moreover, Rayleigh's formula and the uniform ellipticity of the sequence of tensors $K_{T_{n}}^{\star}$ lead to

$$
\lambda_{n}^{1}=\min _{\varphi \neq 0, \varphi \in H_{0}^{1}} \frac{\int_{\Omega} K_{T_{n}}^{\star} \nabla \varphi \cdot \nabla \varphi}{\|\varphi\|_{L_{\beta_{n}^{\star}}^{2}(\Omega)}^{2}} \geq \frac{k_{1}}{\beta_{2}} \min _{\varphi \neq 0, \varphi \in H_{0}^{1}} \frac{\int_{\Omega} \nabla \varphi \cdot \nabla \varphi}{\|\varphi\|_{L^{2}(\Omega)}^{2}}=\frac{k_{1}}{\beta_{2}} \lambda_{1},
$$

where $\lambda_{1}>0$ is the first eigenvalue of the (Dirichlet) Laplacian. This completes the proof of (3.4).

Next, we decompose

$$
\frac{1}{T_{n}} \int_{0}^{T_{n}} \int_{\Omega} K_{T_{n}}^{\star} \nabla u_{n} \cdot \nabla u_{n} d x d t-\int_{\Omega} K_{T_{\infty}}^{\star} \nabla \bar{u}_{\infty} \cdot \nabla \bar{u}_{\infty} d x=I_{1}^{n}+I_{2}^{n}
$$

where

$$
I_{1}^{n}=\frac{1}{T_{n}} \int_{0}^{T_{n}} \int_{\Omega} K_{T_{n}}^{\star} \nabla u_{n}(t, x) \cdot \nabla u_{n}(t, x) d x d t-\int_{\Omega} K_{T_{n}}^{\star} \nabla \bar{u}_{n}(x) \cdot \nabla \bar{u}_{n}(x) d x
$$

and

$$
I_{2}^{n}=\int_{\Omega} K_{T_{n}}^{\star} \nabla \bar{u}_{n}(x) \cdot \nabla \bar{u}_{n}(x) d x-\int_{\Omega} K_{T_{\infty}}^{\star}(x) \nabla \bar{u}_{\infty}(x) \cdot \nabla \bar{u}_{\infty}(x) d x .
$$

Using the weak form of (3.5), multiplying the heat equation (3.1) by $u_{n}(t, x)$ and integrating by parts yield

$$
I_{1}^{n}=\frac{1}{2} \frac{1}{T_{n}} \int_{\Omega} \beta_{n}^{\star}\left(u_{0}^{2}(x)-u_{n}^{2}\left(T_{n}, x\right)\right) d x+\frac{1}{T_{n}} \int_{0}^{T_{n}} \int_{\Omega} f(x)\left(u_{n}(t, x)-\bar{u}_{n}(x)\right) d x d t .
$$

By (3.4) and the boundedness of $\left\|\bar{u}_{n}\right\|_{L^{2}(\Omega)}$, the first term in the right-hand side of this expression converges to zero as $T_{n} \rightarrow \infty$. Using once again (3.4) and the CauchySchwartz inequality,

$$
\begin{aligned}
\left|\frac{1}{T_{n}} \int_{0}^{T_{n}} \int_{\Omega} f(x)\left(u_{n}(t, x)-\bar{u}_{n}(x)\right) d x d t\right| & \leq\|f\|_{L^{2}(\Omega)} \frac{1}{T_{n}} \int_{0}^{T_{n}}\left\|u_{n}(t)-\bar{u}_{n}\right\|_{L^{2}(\Omega)} d t \\
& \leq\|f\|_{L^{2}(\Omega)} \frac{1}{T_{n}} \int_{0}^{T_{n}} C_{1} e^{-C_{2} t} d t \\
& \rightarrow 0 \quad \text { as } T_{n} \rightarrow \infty .
\end{aligned}
$$

This proves that $I_{1}^{n} \rightarrow 0$ as $n \rightarrow \infty$. The fact that $I_{2}^{n}$ also converges to zero as $n \rightarrow \infty$ is a direct consequence of the weak forms of the elliptic systems (3.3) and (3.5) and the convergence $K_{T_{n}}^{\star} \stackrel{\mathrm{H}}{\rightarrow} K_{T_{\infty}}^{\star}$. 
THEOREM 3.2. If $\left(\theta_{T_{n}}, K_{T_{n}}^{\star}\right)$ is an optimal solution of $\left(R P_{T_{n}}\right)$, then any weak limit $\left(\theta_{T_{\infty}}, K_{T_{\infty}}^{\star}\right)$ of a converging subsequence of $\left(\theta_{T_{n}}, K_{T_{n}}^{\star}\right)$ is an optimal solution of $\left(R P_{\infty}\right)$.

Proof. We argue by contradiction. Assume that $\left(\theta_{T_{\infty}}, K_{T_{\infty}}^{\star}\right)$ is not a solution of $\left(\mathrm{RP}_{\infty}\right)$. Then, there exists another $\left(\widehat{\theta}, \widehat{K}^{\star}\right) \in \mathbf{R D}$ and $\varepsilon>0$ such that

$$
\int_{\Omega} K_{T_{\infty}}^{\star}(x) \nabla \bar{u}_{\infty}(x) \cdot \nabla \bar{u}_{\infty}(x) d x=\int_{\Omega} \widehat{K}^{\star}(x) \nabla \widehat{u}(x) \cdot \nabla \widehat{u}(x) d x+\varepsilon,
$$

where $\widehat{u}(x)$ is the solution of the elliptic equation with conductivity $\widehat{K}^{\star}$. By (3.2), there exists $n_{0} \in \mathbb{N}$ such that for all $n \geq n_{0}$

$\frac{1}{T_{n}} \int_{0}^{T_{n}} \int_{\Omega} K_{T_{n}}^{\star}(x) \nabla u_{n}(t, x) \cdot \nabla u_{n}(t, x) d x>\int_{\Omega} K_{T_{\infty}}^{\star}(x) \nabla \bar{u}_{\infty}(x) \cdot \nabla \bar{u}_{\infty}(x) d x-\frac{\varepsilon}{3}$.

Now let $u(t, x)$ solve

$$
\left\{\begin{array}{lll}
\widehat{\beta}^{\star}(x) u^{\prime}(t, x)-\operatorname{div}\left(\widehat{K}^{\star}(x) \nabla u(t, x)\right)=f(x) & \text { in } \quad(0, T) \times \Omega \\
u=0 & \text { on } \quad(0, T) \times \partial \Omega \\
u(0, x)=u_{0}(x) & \text { in } \Omega,
\end{array}\right.
$$

with $\widehat{\beta}^{\star}(x)=\widehat{\theta}(x) \beta_{1}+(1-\widehat{\theta}(x)) \beta_{2}$. Then, multiplying this equation by $u(t, x)$ and integrating by parts, we get the convergence

$$
\lim _{n \rightarrow \infty} \frac{1}{T_{n}} \int_{0}^{T_{n}} \int_{\Omega} \widehat{K}^{\star}(x) \nabla u(t, x) \cdot \nabla u(t, x) d x d t=\int_{\Omega} \widehat{K}^{\star}(x) \nabla \widehat{u}(x) \cdot \nabla \widehat{u}(x) d x .
$$

Therefore, there exists $n_{1} \in \mathbb{N}$ such that for all $n \geq n_{1}$

$$
\frac{1}{T_{n}} \int_{0}^{T_{n}} \int_{\Omega} \widehat{K}^{\star}(x) \nabla u(t, x) \cdot \nabla u(t, x) d x d t<\int_{\Omega} \widehat{K}^{\star}(x) \nabla \widehat{u}(x) \cdot \nabla \widehat{u}(x) d x+\frac{\varepsilon}{3} .
$$

Hence, for $n \geq \max \left(n_{0}, n_{1}\right)$ we have

$$
\begin{aligned}
\frac{1}{T_{n}} \int_{0}^{T_{n}} \int_{\Omega} \widehat{K}^{\star} \nabla u \cdot \nabla u d x d t & <\int_{\Omega} K_{T_{\infty}}^{\star} \nabla \bar{u}_{\infty} \cdot \nabla \bar{u}_{\infty} d x-\varepsilon+\frac{\varepsilon}{3} \\
& <\frac{1}{T_{n}} \int_{0}^{T_{n}} \int_{\Omega} K_{T_{n}}^{\star} \nabla u_{n} \cdot \nabla u_{n} d x d t-\frac{\varepsilon}{3}
\end{aligned}
$$

which contradicts the fact that $\left(\theta_{T_{n}}, K_{T_{n}}^{\star}\right)$ is an optimal solution of $\left(\mathrm{RP}_{T_{n}}\right)$.

REMARK 3.3. In the non-composite region for the optimal solution $\left(\theta_{T_{\infty}}, K_{T_{\infty}}^{\star}\right)$, i.e., both in the set of points $x \in \Omega$ such that $\theta_{T_{\infty}}(x)=0$ or $\theta_{T_{\infty}}(x)=1$, the convergence of the optimal densities $\theta_{T_{n}}$ towards $\theta_{T_{\infty}}$ is in fact a strong convergence in $L^{p}$ for any $1 \leq p<\infty$. Indeed, this is a consequence of the following general result: if a sequence of functions $f_{n}$ satisfies: (a) $0 \leq f_{n} \leq C$, with $C$ a constant, and (b) $f_{n} \rightarrow 0$ weak $-\star$ in $L^{\infty}(\Omega)$, then $f_{n} \rightarrow 0$ strongly in $L^{p}(\Omega)$ for any $1 \leq p<\infty$. Notice that from the estimate $f_{n}^{2} \leq C f_{n}$ we deduce that $\left\|f_{n}\right\|_{L^{2}} \rightarrow 0$. By interpolation, the same holds true for any $L^{p}-$ norm, with $1 \leq p<\infty$. The claim on the densities $\theta_{T_{n}}$ and $\theta_{T_{\infty}}$ just follows by applying this result to $f_{n}=\theta_{T_{n}}$ on the set where $\theta_{T_{\infty}}=0$ and to $f_{n}=1-\theta_{T_{n}}$ on the set where $\theta_{T_{\infty}}=1$. 
4. Optimality conditions for the parabolic case. This section is devoted to the study of the necessary optimality conditions that a minimizer $\left(\theta_{T}, K_{T}^{\star}\right)$ of $\left(\mathrm{RP}_{T}\right)$ must satisfy. We follow the same ideas as in the elliptic and hyperbolic cases (see $[1,2,21])$.

In order to take into account the volume constraint on $\theta$, we consider for any $l \in \mathbb{R}$ and $\left(\theta, K^{\star}\right) \in \mathbf{R D}$ the augmented function

$$
\bar{J}_{T}^{\star}\left(\theta, K^{\star}\right)=\frac{1}{T} \int_{0}^{T} \int_{\Omega} K^{\star} \nabla u \cdot \nabla u d x d t+l \int_{\Omega} \theta(x) d x .
$$

Then, we have :

THEOREM 4.1. The objective function $\bar{J}_{T}^{\star}\left(\theta, K^{\star}\right)$ is Gâteaux differentiable on the space of admissible relaxed designs $\boldsymbol{R D}$ and

$$
\begin{aligned}
\delta \bar{J}_{T}^{\star}\left(\theta, K^{\star}\right) & =\int_{\Omega}\left[l-\frac{2\left(\beta_{2}-\beta_{1}\right)}{T} \int_{0}^{T} u^{\prime} p d t\right] \delta \theta d x \\
& +\frac{1}{T} \int_{0}^{T} \int_{\Omega} \delta K^{\star} \nabla u \cdot(2 \nabla p+\nabla u) d x d t
\end{aligned}
$$

where $\delta \theta$ and $\delta K^{\star}$ are admissible increments in $\boldsymbol{R D}$ and $p$ the solution of the adjoint equation

$$
\left\{\begin{array}{lll}
-\beta^{\star} p^{\prime}-\operatorname{div}\left(K^{\star} \nabla p\right)=\operatorname{div}\left(K^{\star} \nabla u\right) & \text { in } & (0, T) \times \Omega \\
p=0 & \text { on } & (0, T) \times \partial \Omega \\
p(T)=0 & \text { in } & \Omega .
\end{array}\right.
$$

Consequently, if $\left(\theta_{T}, K_{T}^{\star}\right)$ is a minimizer of $\bar{J}_{T}^{\star}$, it must satisfy $\delta \bar{J}_{T}^{\star}\left(\theta_{T}, K_{T}^{\star}\right) \geq 0$ for any admissible increments $\delta \theta, \delta K^{\star}$.

Proof. The proof is standard. A direct derivation shows that

$\delta \bar{J}_{T}^{\star}\left(\theta, K^{\star}\right)=l \int_{\Omega} \delta \theta d x+\frac{1}{T}\left[\int_{0}^{T} \int_{\Omega} \delta K^{\star} \nabla u \cdot \nabla u d x d t+2 \int_{0}^{T} \int_{\Omega} K^{\star} \nabla u \cdot \nabla \delta u d x d t\right]$,

where $\delta u$ is the increment in the solution $u$ caused by the increment $\delta \theta$ and $\delta K^{\star}$. By differentiating the state equation $(2.3)$, we obtain that the increment $\delta u$ solves the problem

$$
\left\{\begin{array}{lll}
\beta^{\star}(\delta u)^{\prime}-\operatorname{div}\left(K^{\star} \nabla \delta u\right)=\left(\beta_{2}-\beta_{1}\right) u^{\prime} \delta \theta+\operatorname{div}\left(\delta K^{\star} \nabla u\right) & \text { in }(0, T) \times \Omega, \\
\delta u=0 & \text { on }(0, T) \times \partial \Omega \\
\delta u(0)=0 & \text { in } \Omega .
\end{array}\right.
$$

The introduction of the adjoint state allows one to eliminate the increment $\delta u$. Let us first remark that (4.3) admits a unique solution $p \in L^{2}\left((0, T) ; H_{0}^{1}(\Omega)\right)$ since the right hand side of $(4.3), \operatorname{div}\left(K^{\star} \nabla u\right)$, belongs to $L^{2}\left((0, T) ; H^{-1}(\Omega)\right)$. Recall that $f \in$ $L^{2}((0, T) \times \Omega)$ and $u_{0} \in L^{2}(\Omega)$ imply that $u \in L^{2}\left((0, T) ; H_{0}^{1}(\Omega)\right) \cap C\left([0, T] ; L^{2}(\Omega)\right)$ and $u^{\prime} \in L^{2}\left((0, T) ; H^{-1}(\Omega)\right)$ (see [7, p. 244] for more details). In particular, it implies that the right hand side of (4.2) is well defined. Multiplying equations (4.4) by $p$ and (4.3) by $\delta u$, then integrating by parts, yields

$$
\int_{0}^{T} \int_{\Omega} K^{\star} \nabla u \cdot \nabla \delta u d x d t=-\int_{0}^{T} \int_{\Omega}\left(\beta_{2}-\beta_{1}\right) u^{\prime} p \delta \theta d x d t+\int_{0}^{T} \int_{\Omega} \delta K^{\star} \nabla u \cdot \nabla p d x d t
$$

and finally gives (4.2). 
This result allows us to prove that an optimal tensor can always be found in the class of sequential laminates of rank at most $N$ (see [1] for a precise definition). We first introduce the following definition:

Definition 4.2. Let $\left(\theta, K^{\star}\right) \in \boldsymbol{R} \boldsymbol{D}$ satisfy the optimality condition $\delta \bar{J}_{T}^{\star}\left(\theta, K^{\star}\right) \geq$ 0 . For any fixed $T>0$, we introduce the symmetric matrix of order $N$

$$
M_{T}=-\frac{1}{T} \int_{0}^{T} \nabla u \odot(2 \nabla p+\nabla u) d t
$$

where $\odot$ denotes the symmetrized tensor product of two vectors, with entries

$$
\left(M_{T}\right)_{i j}=-\frac{1}{2 T} \int_{0}^{T}\left[(\nabla u)_{i}(2 \nabla p+\nabla u)_{j}+(\nabla u)_{j}(2 \nabla p+\nabla u)_{i}\right] d t,
$$

where $u$ and $p$ are its associated state and adjoint state, respectively.

REMARK 4.3. The matrix $M_{T}$ belongs to $L^{1}(\Omega)$ since, as already explained in the proof of Theorem 4.1, $\nabla u$ and $\nabla p$ belong to $L^{2}((0, T) \times \Omega)$.

THEOREM 4.4 (Order of lamination). Let $\left(\theta_{T}, K_{T}^{\star}\right) \in \boldsymbol{R} \boldsymbol{D}$ be a minimizer of (4.1) and let $u$ and $p$ be its associated state and adjoint state, respectively. Then, at the points where $M_{T}$ does not vanish, the effective tensor $K_{T}^{\star}$ belongs to the boundary of the set $G_{\theta_{T}}$ and thus corresponds to a sequential laminate of rank at most $N$ with lamination directions given by the eigenvectors of the matrix $M_{T}$, defined by (4.5). It is also a maximizer of the function

$$
f\left(\theta_{T}, M_{T}\right)=K_{T}^{\star}: M_{T}=\max _{K^{0} \in G_{\theta_{T}}} K^{0}: M_{T} .
$$

Moreover, the function $\theta_{T} \longmapsto f\left(\theta_{T}, M_{T}\right)$ is $C^{1}([0,1])$ and the optimal density $\theta_{T}$ satisfies

$$
\left\{\begin{array}{lll}
\theta_{T}(x)=0 & \text { if and only if } & Q_{T}(x)>0 \\
\theta_{T}(x)=1 & \text { if and only if } & Q_{T}(x)<0 \\
0 \leq \theta_{T}(x) \leq 1 & \text { if } & Q_{T}(x)=0
\end{array}\right.
$$

and $Q_{T}(x)=0$ if $0<\theta_{T}(x)<1$, where $Q_{T}$ is given by

$$
Q_{T}(x)=l-2\left(\beta_{2}-\beta_{1}\right) \frac{1}{T} \int_{0}^{T} u^{\prime} p d t+\frac{\partial f}{\partial \theta}\left(\theta_{T}, M_{T}\right) .
$$

At the points where $M_{T}$ vanishes, if we assume in addition that $\beta_{1}=\beta_{2}$, then there exists another minimizer $\left(\tilde{\theta}_{T}, K_{T}^{\star}\right)$ of (4.1) with the same state $u$ and adjoint state $p$ which satisfies the above properties with $\theta_{T}$ replaced by $\tilde{\theta}_{T}$, namely $K_{T}^{\star}$ is a rank- $N$ sequential laminate which belongs to the boundary of $G_{\tilde{\theta}_{T}}$.

In (4.6) the notation $A: B$ stands for the full contraction of matrices $A$ and $B$.

REMARK 4.5. In the previous theorem, at the points where $M_{T}$ vanishes, it is necessary to change the optimal density $\theta_{T}$ (but not the optimal tensor $K_{T}^{\star}$ ). Such a trick was first devised by U. Raitums [17]. The main interest of Theorem 4.4 is that one can restrict the minimization of (4.1) to the subclass of effective tensors which are given by the simple and explicit formula of sequential laminates of rank $N$, at most, with orthogonal lamination directions (see Chapter 3 of [1]).

Proof. The proof follows the same lines as in the elliptic case (see [1, Th. 3.2.14]), except for the new case when $M_{T}=0$. We fix $\theta_{T}$ and consider the path $K_{T}^{\star}(s)=$ 
$s K^{0}+(1-s) K_{T}^{\star}$ for any $K^{0} \in G_{\theta_{T}}, 0 \leq s \leq 1$ and for $K_{T}^{\star}$ an optimal tensor for $\left(\mathrm{RP}_{T}\right)$. Consequently, $\delta \theta=0$ and $\delta K_{T}^{\star}=K^{0}-K_{T}^{\star}$. Taking into account the optimality condition $\delta \bar{J}_{T}^{\star}\left(\theta_{T}, K_{T}^{\star}\right) \geq 0$ and the relation (4.2), we conclude that

$$
\int_{\Omega} K_{T}^{\star}: M_{T} d x \geq \int_{\Omega} K^{0}: M_{T} d x \quad \forall K^{0} \in G_{\theta_{T}} .
$$

By localization, (4.9) is equivalent a.e. $x \in \Omega$ to the following characterization of the optimal tensor $K_{T}^{\star}$

$$
K_{T}^{\star}: M_{T}=\max _{K^{0} \in G_{\theta_{T}}} K^{0}: M_{T}, \quad \text { a.e. } \quad x \in \Omega .
$$

It is known that the optimal tensor $K_{T}^{\star}$ of (4.10) must be simultaneously diagonalizable with $M_{T}$. Consequently, if $\left(e_{j}\right)_{1 \leq j \leq N}$ is a basis of eigenvectors of $M_{T}$ with associated eigenvalues $\left(\mu_{j}\right)_{1 \leq j \leq N}$, one can restrict the maximization in (4.10) to those tensors $K^{0}$ that share the same eigenvectors with eigenvalues $\left(\lambda_{j}\right)$ and (4.10) is equivalent to

$$
K_{T}^{\star}: M_{T}=\max _{\left(\lambda_{j}\right) \in G_{\theta_{T}}} \sum_{j=1}^{N} \lambda_{j} \mu_{j} .
$$

Assume that $x \in \Omega$ is such that $M_{T}(x) \neq 0$. Since the cost function in (4.11) is linear and the set $G_{\theta_{T}}$ convex, the solution of this problem belongs to the boundary of $G_{\theta_{T}}$. This implies (see the proof of [1, Ths. 2.2.13 and 3.2.14]) that the optimal $K_{T}^{\star}$ corresponds to a sequential laminate of rank at most $N$ with lamination directions given by the eigenvectors of $M_{T}$.

Let us now consider the points $x \in \Omega$ such that $M_{T}(x)=0$. If the optimal tensor $K_{T}^{\star}$ happens to belong to the boundary of the set $G_{\theta_{T}}$, defined by (2.1), we are done since the boundary of $G_{\theta}$ is made of sequential laminates of rank at most $N$. We now restrict our attention to the case when $M_{T}(x)=0$ and $K_{T}^{\star}$ belongs to the interior of $G_{\theta_{T}}$ : we denote by $\omega \subset \Omega$ the subset of such points where we shall modify the optimal density $\theta_{T}$. Since $K_{T}^{\star}$ does not belong to the boundary of $G_{\theta_{T}}$, at a point $x \in \omega$, if we denote by $\left(\lambda_{j}^{\star}\right)_{1 \leq j \leq N}$ the eigenvalues of $K_{T}^{\star}$, they satisfy strict inequalities in all inequalities of (2.1). (In particular it excludes the special case $\theta_{T}=0$ or 1.) The lower bounds of $G_{\theta}$ are

$$
\lambda_{\theta}^{-} \leq \lambda_{j}^{\star}, \quad \sum_{j=1}^{N} \frac{1}{\lambda_{j}^{\star}-k_{1}} \leq h_{\theta}^{-}=\frac{1}{\lambda_{\theta}^{-}-k_{1}}+\frac{N-1}{\lambda_{\theta}^{+}-k_{1}},
$$

which are (geometrically) strictly decreasing functions of $\theta$ (more precisely, $\theta \rightarrow h_{\theta}^{-}$ is increasing and $\theta \rightarrow \lambda_{\theta}^{-}$is decreasing). See Fig. 4.1 for a better understanding of what we mean by saying that the set $G_{\theta}$ (in particular, its lower bounds (4.12)) is geometrically decreasing as a function of $\theta$.

Therefore, there exists a value $0<\theta^{-}<\theta$ such that the lower bound (4.12) is saturated for $\theta^{-}$(namely, one of the inequalities in (4.12) is actually an equality) and thus $K_{T}^{\star}$ belongs to the "lower" boundary of $G_{\theta^{-}}$. Similarly, the upper bounds of $G_{\theta}$ are

$$
\lambda_{j}^{\star} \leq \lambda_{\theta}^{+}, \quad \sum_{j=1}^{N} \frac{1}{k_{2}-\lambda_{j}^{\star}} \leq h_{\theta}^{+}=\frac{1}{k_{2}-\lambda_{\theta}^{-}}+\frac{N-1}{k_{2}-\lambda_{\theta}^{+}},
$$




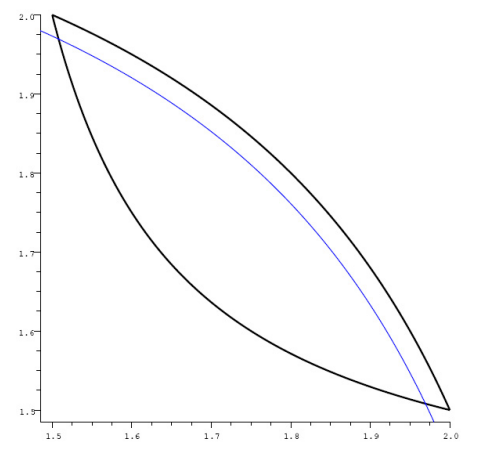

FIG. 4.1. Picture of the set $G_{\theta}$ for $N=2, \theta=0.5, k_{1}=1$ and $k_{2}=3$. To emphasize the geometrical decreasing character of this set we have also plotted the upper bound for $\theta=0.51$ with the same $k_{1}$ and $k_{2}$.

which are (geometrically) strictly decreasing functions of $\theta$ (i.e., $\theta \rightarrow h_{\theta}^{+}$is decreasing and $\theta \rightarrow \lambda_{\theta}^{+}$is decreasing). Therefore, there exists a value $\theta<\theta^{+}<1$ such that the upper bound (4.13) is saturated for $\theta^{+}$(namely, one of the inequalities in (4.13) is actually an equality) and thus $K_{T}^{\star}$ belongs to the "upper" boundary of $G_{\theta^{+}}$.

Overall, we have proved that, for each $x \in \omega$, there exist two densities $0<\theta^{-}(x)<$ $\theta_{T}(x)<\theta^{+}(x)<1$ such that, $K_{T}^{\star}$ belongs simultaneously to the "upper" boundary of $G_{\theta^{+}}$and to the "lower" boundary of $G_{\theta^{-}}$. We can divide the subset $\omega$ in two parts $\omega^{+}$and $\omega^{-}$where the density $\theta_{T}$ is changed in

$$
\tilde{\theta}_{T}(x)= \begin{cases}\theta^{+}(x) & \text { if } x \in \omega^{+} \\ \theta^{-}(x) & \text { if } x \in \omega^{-} \\ \theta_{T}(x) & \text { otherwise. }\end{cases}
$$

Of course, we can choose $\omega^{+}$and $\omega^{-}$in such a way that the volume constraint is kept, i.e., $\int_{\Omega} \tilde{\theta}_{T} d x=\int_{\Omega} \theta_{T} d x$. Remark that it is not necessary to change $K_{T}^{\star}$ which belongs to both sets $G_{\theta_{T}}$ and $G_{\tilde{\theta}_{T}}$. The point is that, by construction, $K_{T}^{\star}$ lies on the boundary of $G_{\tilde{\theta}_{T}}$ and is therefore a sequential laminate of rank at most $N$ with lamination directions given by the eigenvectors of $M_{T}$. Notice that since $K_{T}^{\star}$ does not change and $\beta_{1}=\beta_{2}, u$ and $p$ are the same and so is the value of the cost function.

Next, we consider a smooth path $\left(\theta(s), K^{\star}(s)\right)_{0<s<1} \in \mathbf{R D}$ in the space of admissible relaxed designs such that at $s=0$ it coincides with a minimizer, say $\left(\theta_{T}, K_{T}^{\star}\right)$, of $\left(\mathrm{RP}_{T}\right)$. We further ask that, for each $s, K^{\star}(s)$ is optimal for the density $\theta(s)$, in the sense that

$$
K^{\star}(s): M_{T}=f\left(\theta(s), M_{T}\right)=\max _{K^{0} \in G_{\theta}} K^{0}: M_{T}
$$

where $M_{T}$ is defined by (4.5). Such a function $f$ is known to be differentiable with respect to $\theta$ (see [1, Th. 3.2.14]) and, differentiating (4.14) with respect to $s$, we get

$$
\delta K^{\star}: M_{T}=\frac{\partial f}{\partial \theta}\left(\theta, M_{T}\right) \delta \theta .
$$

Replacing this expression in (4.2) we conclude that the optimal $\left(\theta_{T}, K_{T}^{\star}\right)$ satisfies

$$
\delta \bar{J}_{T}^{\star}\left(\theta_{T}, K_{T}^{\star}\right)=\int_{\Omega} Q_{T}(x) \delta \theta d x \geq 0
$$


for any admissible increment $\delta \theta$, where $Q_{T}$ is defined by (4.8). By localization we thus obtain (4.7).

REMARK 4.6. The argument we have used in the previous theorem to deal with the case $M_{T}(x)=0$ does not extend to the situation in which $\beta_{1} \neq \beta_{2}$. The reason is that if $K_{T}^{\star}$ does not change but $\beta_{1} \neq \beta_{2}$, then the corresponding $u$ and $p$ do change and therefore we cannot ensure that the associated value for the cost function is optimal. Later on (Theorem 5.2) we provide a sufficient condition on the source term $f$ which enables us to conclude, for $T$ large enough, about the order of lamination without constraint on the values of the positive constants $\beta_{1}$ and $\beta_{2}$.

REMARK 4.7. The proof of Theorem 4.4 can immediately be extended to a multiple load case (static or parabolic). Our result is reminiscent, but different, of a recent one in [2]. In this reference, concerned with two-phase optimal design in the static conductivity case, the authors consider $m$ state equations with $m<N$ and prove that an optimal effective tensor can always be found among sequential laminates with matrix material $k_{1}$ of rank at most $m$. Our result has no such restriction on the number of state equations but, on the other hand, the matrix material can be either $k_{1}$ or $k_{2}$.

An optimality criteria method, based on the above necessary optimality conditions, may be implemented for solving numerically the relaxed problem $\left(\mathrm{RP}_{T}\right)$. Such an algorithm reads as follows:

- Initialization: take initial values $\left(\theta^{0}, K^{0}\right)$ in $\mathbf{R D}$ and $l^{0}$ in $\mathbb{R}$ for the density, homogenized tensor and Lagrange multiplier, respectively. For instance, $\theta^{0} \equiv$ $L, K^{0}=\operatorname{diag}\left(\lambda_{\theta^{0}}^{-}, \lambda_{\theta^{0}}^{+}, \cdots, \lambda_{\theta^{0}}^{+}\right)$and $l^{0}=0$.

- For $n \geq 0$, iteration until convergence as follows:

1. Compute the state $u^{n}$ and the adjoint state $p^{n}$ by solving (2.3) and (4.3) respectively.

2. Compute in $\Omega$, the matrix $M_{T}^{n}$ as given by (4.5). Then, the function $f\left(\theta^{n}, M_{T}^{n}\right)$ in (4.6). For $N=2$, this can be done explicitly (see [1, Lemma 3.2.17, p. 231] and Lemma 4.9 below).

3. Compute the function $Q_{T}^{n}(x)$ as defined in (4.8) and then, by (4.7), compute a new density $\widetilde{\theta}^{n+1}$ which depends on the Lagrange multiplier $l^{n}$ through (4.8). Since a priori $\widetilde{\theta}^{n+1}$ does not necessarily satisfy the volume constraint $\|\theta\|_{L^{1}(\Omega)}=L|\Omega|$, we determine the optimal multiplier $l^{n+1}$ so that the corresponding density $\theta^{n+1}$ satisfies this constraint. This is easily done using the monotony of the (possibly multi-valued) function

$$
\Theta(l)=\int_{\Omega} \theta_{l}(x) d x
$$

$\theta_{l}$ being the optimal density of $\left(\mathrm{RP}_{T}\right)$ with multiplier $l$ (see Lemma 4.8 bellow).

4. Finally, solve (4.11) to get an update tensor $K^{n+1}$.

For the sake of completeness we recall a result on the monotonicity of the volume constraint with respect to the Lagrange multiplier.

LEMmA 4.8. The function $\Theta:[0,1] \rightarrow \mathbb{R}$, defined by (4.15), is non-increasing.

Proof. Assume that $l<l^{\prime}$ and take any minimizer $\left(\theta_{l}, K_{l}^{\star}\right)$ (respectively $\left(\theta_{l^{\prime}}, K_{l^{\prime}}^{\star}\right)$ 
of (4.1). From the optimality of $\left(\theta_{l^{\prime}}, K_{l^{\prime}}^{\star}\right)$, we have

$$
\int_{0}^{T} \int_{\Omega} K_{l^{\prime}}^{\star} \nabla u_{l^{\prime}} \cdot \nabla u_{l^{\prime}} d x d t+l^{\prime} \Theta\left(l^{\prime}\right) \leq \int_{0}^{T} \int_{\Omega} K_{l}^{\star} \nabla u_{l} \cdot \nabla u_{l} d x d t+l^{\prime} \Theta(l)
$$

which is equivalent to

$$
\begin{aligned}
\int_{0}^{T} \int_{\Omega} K_{l^{\prime}}^{\star} \nabla u_{l^{\prime}} \cdot \nabla u_{l^{\prime}} d x d t+l \Theta\left(l^{\prime}\right) \leq & \int_{0}^{T} \int_{\Omega} K_{l}^{\star} \nabla u_{l} \cdot \nabla u_{l} d x d t+l \Theta(l) \\
& +\left(l^{\prime}-l\right)\left(\Theta(l)-\Theta\left(l^{\prime}\right)\right) .
\end{aligned}
$$

Since the first two terms in the right-hand side of this expression give an optimal value, the third term must be non-negative, i.e. $\Theta(l) \geq \Theta\left(l^{\prime}\right)$.

In two space dimensions, $N=2$, the function $f\left(\theta_{T}, M_{T}\right)$, introduced in Theorem 4.4, can be explicitly computed (see [1, Lemma 3.2.17] and [22] for a similar computation in the case $N=3$ ). As a byproduct it gives the precise order of lamination of the optimal microstructure in the optimization problem (4.11).

Lemma 4.9. For any $T>0$, we note by $\mu_{1}^{T} \leq \mu_{2}^{T}$ the eigenvalues of the matrix $M_{T}$ of order $N=2$ defined by (4.5) and by $v_{1}^{T} \leq v_{2}^{T}$ those of the matrix $K^{0}$. The solution of the linear problem

$$
\max _{K^{0} \in G_{\theta_{T}}} K^{0}: M_{T}=\max _{\left(v_{1}^{T}, v_{2}^{T}\right) \in G_{\theta_{T}}} v_{1}^{T} \mu_{1}^{T}+v_{2}^{T} \mu_{2}^{T}
$$

is given by

$$
\left\{\begin{aligned}
&\left(v_{1}^{T}, v_{2}^{T}\right)=\left(k_{2}, k_{2}\right)+\frac{\sqrt{\mu_{1}^{T}}+\sqrt{\mu_{2}^{T}}}{\left(\lambda_{\theta_{T}}^{+}-k_{2}\right)^{-1}+\left(\lambda_{\theta_{T}}^{-}-k_{2}\right)^{-1}}\left(\frac{1}{\sqrt{\mu_{1}^{T}}}, \frac{1}{\sqrt{\mu_{2}^{T}}}\right) \\
& \text { if } \quad \mu_{1}^{T} \geq 0 \quad \text { and } \sqrt{\mu_{1}^{T}}\left(k_{2}-\lambda_{\theta_{T}}^{-}\right)>\sqrt{\mu_{2}^{T}}\left(k_{2}-\lambda_{\theta_{T}}^{+}\right) \\
&\left(v_{1}^{T}, v_{2}^{T}\right)=\left(k_{1}, k_{1}\right)+\frac{\sqrt{-\mu_{1}^{T}}+\sqrt{-\mu_{2}^{T}}}{\left(\lambda_{\theta_{T}}^{+}-k_{1}\right)^{-1}+\left(\lambda_{\theta_{T}}^{-}-k_{1}\right)^{-1}}\left(\frac{1}{\sqrt{-\mu_{1}^{T}}}, \frac{1}{\sqrt{-\mu_{2}^{T}}}\right) \\
& \text { if } \quad \mu_{2}^{T} \leq 0 \quad \text { and } \sqrt{-\mu_{1}^{T}}\left(\lambda_{\theta_{T}}^{-}-k_{1}\right)<\sqrt{-\mu_{2}^{T}}\left(\lambda_{\theta_{T}}^{+}-k_{1}\right) \\
&\left(v_{1}^{T}, v_{2}^{T}\right)=\left(\lambda_{\theta_{T}}^{-}, \lambda_{\theta_{T}}^{+}\right) \quad \text { else. }
\end{aligned}\right.
$$

The first and second regimes in (4.16) correspond to a rank-2 laminate while the third one corresponds to a rank-1 laminate. Notice that the third regime in (4.16) is obtained in particular when $\mu_{1}^{T} \mu_{2}^{T} \leq 0$.

5. Asymptotics of the optimality condition when $T \rightarrow \infty$. From the previous analysis, we know that the optimal design $\left(\theta_{T}, K_{T}^{\star}\right)$ for the parabolic problem $\left(R P_{T}\right)$ - for any finite $T$ - may be recovered by a laminate of rank at most $N$ and degenerates as $T \rightarrow \infty$ to a solution of the elliptic problem $\left(R P_{\infty}\right)$, this latter being recovered by laminates of first order. This section is devoted to the analysis of the asymptotic behavior of the optimality conditions when $T \rightarrow \infty$.

We shall need the following technical result. To make clear the dependence on the space dimension, from now on in this section we denote by $G_{\theta}^{N}$ the space $G_{\theta}$ in dimension $N$. 
Lemma 5.1. Let $M_{n} \in \mathcal{M}_{N}^{s}$ be a sequence of symmetric matrices such that

$$
\lim _{n \rightarrow \infty} M_{n}=M,
$$

with $M$ a rank-one, non-negative matrix. Then there exists $n_{0} \in \mathbb{N}$ such that for $n \geq n_{0}$ any maximizer of

$$
\max _{K^{0} \in G_{\theta}^{N}} K^{0}: M_{n}
$$

is a laminate of rank less than or equal to $N-1$ and at least one maximizer is a rank-one laminate.

Proof. Assume that $0<\theta<1$. Otherwise, there is nothing to prove. Denoting by $\lambda_{n}^{1} \leq \lambda_{n}^{2} \leq \cdots \leq \lambda_{n}^{N}$ the eigenvalues of $M_{n}$, we know that

$$
\begin{aligned}
& \lambda_{n}^{i} \rightarrow 0 \\
& \lambda_{n}^{N} \rightarrow \lambda
\end{aligned} \quad \forall i \in\{1,2, \cdots, N-1\}
$$

where $\lambda$ is the only positive eigenvalue of $M$. Consider first the case $N=2$ and the problem

$$
\max _{K^{0} \in G_{\theta}^{2}} K^{0}: M
$$

Problem (5.4) amounts to find $\left(\mu_{1}, \mu_{2}\right) \in G_{\theta}^{2}$, with $\mu_{1} \leq \mu_{2}$, for which the quantity

$$
\mu_{2} \lambda=c
$$

attains it maximum. Geometrically in the plane $\left(\mu_{1}, \mu_{2}\right),(5.5)$ represents an horizontal line cutting the set $G_{\theta}^{2}$ with the largest possible value of $c$, therefore touching $G_{\theta}^{2}$ at a single point, its upper corner corresponding to a rank-one laminate (see Fig. 4.1 or [1, p. 121]). Thus, (5.4) admits a unique maximizer $\left(\mu_{1}, \mu_{2}\right)=\left(\lambda_{\theta}^{-}, \lambda_{\theta}^{+}\right)$. If we now consider problem (5.2), then the associated line, $\mu_{2} \lambda_{n}^{2}+\mu_{1} \lambda_{n}^{1}=c$, is almost horizontal for large $n$, thanks to the convergence (5.1). Since the tangent slopes at the upper corner of $G_{\theta}^{2}$ are neither zero nor infinity, as in the preceding case, (5.2) has a unique maximizer too which is again a first-order laminate.

Notice also that for $N=2$, problem (5.2) has been solved explicitly in Lemma 4.9. Indeed, since $\left(\lambda_{n}^{1}, \lambda_{n}^{2}\right) \rightarrow(0, \lambda)$ as $n \rightarrow 0$, the inequalities of the first regime in Lemma 4.9, i.e. $\lambda_{n}^{1} \geq 0$ and $\sqrt{\lambda_{n}^{1}}\left(k_{2}-\lambda_{\theta}^{-}\right)>\sqrt{\lambda_{n}^{2}}\left(k_{2}-\lambda_{\theta}^{+}\right)$can not hold simultaneously as soon as $n$ is large enough. The second regime is also excluded since by assumption $\lambda_{n}^{2}$ is positive for large $n$. Therefore, for $n$ large enough, the maximum in (5.4) is reached for $\left(\mu_{1}, \mu_{2}\right)=\left(\lambda_{\theta}^{-}, \lambda_{\theta}^{+}\right)$and corresponds to a rank-1 laminate.

The above geometric argument also works in higher dimensions. The reason is that the tangent planes to any smooth curved surface lying on the boundary of $G_{\theta}^{N}$ are not horizontal (neither vertical by the way) so that the (almost) horizontal planes associated with (5.2), as described above, cannot be tangent to any of the smooth curved part of the boundary of $G_{\theta}^{N}$. The only difference with respect to the twodimensional case is that for $N \geq 3$ the solution of (5.2) may be not unique and optimal laminates of rank higher than one can appear. For instance, in the case $N=3$, it is clear that if $M_{n}$ is horizontal (i.e., $\lambda_{n}^{1}=\lambda_{n}^{2}=0$ ), then every point belonging to the flat surface which contains the points $A$ and $B$ in Figure 5.1 is a solution of (5.2). This face is made of rank-2 laminates. 


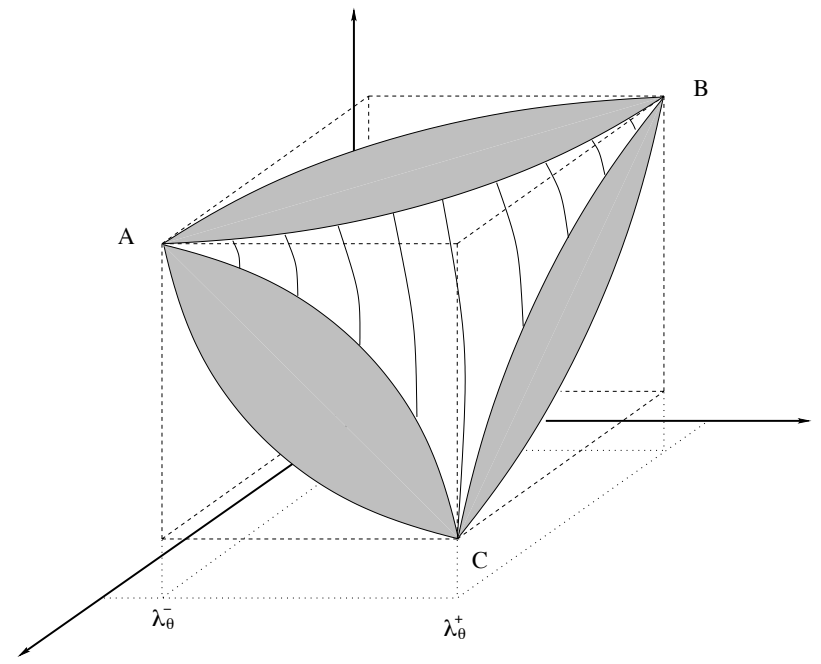

FIG. 5.1. Picture of the set $G_{\theta}^{3}$.

Therefore, in dimension $N$ any maximizer is a sequential laminate of rank at most $N-1$, but there exists at least one maximizer which is a rank-one laminate.

THEOREM 5.2. Let $\left(\theta_{T_{n}}, K_{T_{n}}^{\star}\right)$ be a minimizer of (4.1) and assume that the set of points where $f$ vanishes has zero measure. For a.e. $x \in \Omega$ there exists a positive time $T_{n_{0}}=T_{n_{0}}(x)$ such that for $T_{n} \geq T_{n_{0}}, K_{T_{n}}^{\star}(x)$ is a laminate of rank at most $N-1$.

Proof. We proceed in three steps:

Step 1: energy estimates for the solutions $u(t, x)$ and $p(t, x)$ of (2.3) and (4.3), respectively, for $f=f(x) \in L^{2}(\Omega)$ and $u_{0} \in L^{2}(\Omega)$.

With the change $\tau=\frac{t}{T}$ in the time variable, systems (2.3) and (4.3) transform, respectively, into

$$
\left\{\begin{array}{lll}
\frac{\beta^{\star}(x)}{T} \widetilde{u}^{\prime}(\tau, x)-\operatorname{div}\left(K^{\star}(x) \nabla \widetilde{u}(\tau, x)\right)=f(x) & \text { in } \quad(0,1) \times \Omega \\
\widetilde{u}=0 & \text { on } \quad(0,1) \times \partial \Omega \\
\widetilde{u}(0, x)=u_{0}(x) & \text { in } \quad \Omega
\end{array}\right.
$$

and

$$
\left\{\begin{array}{lll}
-\frac{\beta^{\star}(x)}{T} \widetilde{p}^{\prime}(\tau, x)-\operatorname{div}\left(K^{\star}(x) \nabla \widetilde{p}(\tau, x)\right)=\operatorname{div}\left(K^{\star}(x) \nabla \widetilde{u}(\tau, x)\right) & \text { in } \quad(0,1) \times \Omega \\
\widetilde{p}=0 & \text { on }(0,1) \times \partial \Omega \\
\widetilde{p}(1, x)=0 & \text { in } \quad \Omega,
\end{array}\right.
$$

where $\widetilde{u}(\tau, x)=u(t, x)$ and $\widetilde{p}(\tau, x)=p(t, x)$. The associated matrix $M_{T}$ takes then the form

$$
M_{T}(x)=-\int_{0}^{1} \nabla \widetilde{u}(\tau, x) \odot(2 \nabla \widetilde{p}(\tau, x)+\nabla \widetilde{u}(\tau, x)) d \tau, \quad \text { a. e. } x \in \Omega .
$$

Now let $\bar{u}(x)$ and $\bar{p}(x)$ be the weak solutions of the stationary equations

$$
\left\{\begin{array}{lll}
-\operatorname{div}\left(K^{\star} \nabla \bar{u}\right)=f & \text { in } & \Omega \\
\bar{u}=0 & \text { on } & \partial \Omega
\end{array}\right.
$$


and

$$
\left\{\begin{array}{lll}
-\operatorname{div}\left(K^{\star} \nabla \bar{p}\right)=\operatorname{div}\left(K^{\star} \nabla \bar{u}\right)=-f & \text { in } & \Omega \\
\bar{p}=0 & \text { on } & \partial \Omega,
\end{array}\right.
$$

respectively, so that $\bar{u}=-\bar{p}$. Then, the differences $v(\tau, x)=\widetilde{u}(\tau, x)-\bar{u}(x)$ and $q=\widetilde{p}(\tau, x)-\bar{p}(x)$ solve

$$
\left\{\begin{array}{lll}
\frac{\beta^{\star}(x)}{T} v^{\prime}(\tau, x)-\operatorname{div}\left(K^{\star}(x) \nabla v(\tau, x)\right)=0 & \text { in } & (0,1) \times \Omega \\
v=0 & \text { on } \quad(0,1) \times \partial \Omega \\
v(0, x)=u_{0}(x)-\bar{u}(x) & \text { in } \Omega
\end{array}\right.
$$

and

$$
\left\{\begin{array}{lll}
-\frac{\beta^{\star}(x)}{T} q^{\prime}(\tau, x)-\operatorname{div}\left(K^{\star}(x) \nabla q(\tau, x)\right)=0 & \text { in } & (0,1) \times \Omega \\
q=0 & \text { on } & (0,1) \times \partial \Omega \\
q(1, x)=\bar{u}(x) & \text { in } & \Omega .
\end{array}\right.
$$

Multiplying the PDE in system (5.9) by $v$ and integrating by parts we get the identity

$$
\frac{1}{2 T} \int_{\Omega} \beta^{\star}(x) v^{2}(1) d x+\int_{0}^{1} \int_{\Omega} K^{\star} \nabla v \cdot \nabla v d x d \tau=\frac{1}{2 T} \int_{\Omega} \beta^{\star}(x)\left(u_{0}(x)-\bar{u}(x)\right)^{2} d x .
$$

Taking into account the ellipticity assumption on the tensor $K^{\star}$ and the Poincaré inequality one easily obtains

$$
\|v\|_{L^{2}\left((0,1) \times H_{0}^{1}(\Omega)\right)} \leq \frac{C}{\sqrt{T}} .
$$

Here and in the sequel, $C$ stands for a positive constant which may change from line to line, but which is independent of $T$.

Similarly,

$$
\|q\|_{L^{2}\left((0,1) \times H_{0}^{1}(\Omega)\right)} \leq \frac{C}{\sqrt{T}} .
$$

Step 2: consider the matrix $M_{\infty}=\nabla \bar{u} \odot \nabla \bar{u}$. Then we have the estimate

$$
\int_{\Omega}\left|M_{T}(x)-M_{\infty}(x)\right| d x \leq \frac{C}{T} .
$$

Indeed, after some simple algebra we have

$$
\begin{aligned}
M_{T}-M_{\infty} & =-\int_{0}^{1}[\nabla(\bar{u}+v) \odot \nabla(2(\bar{p}+q)+\bar{u}+v)+\nabla \bar{u} \odot \nabla \bar{u}] d \tau \\
& =-\nabla \bar{u} \odot \nabla\left(2 \int_{0}^{1} q d \tau\right)-\int_{0}^{1} \nabla v \odot \nabla(v+2 q) d \tau .
\end{aligned}
$$

Since $\int_{0}^{1} q d \tau$ satisfies

$$
\left\{\begin{array}{lll}
-\operatorname{div}\left[K^{\star} \nabla\left(\int_{0}^{1} q d \tau\right)\right]=\frac{\beta^{\star}}{T} \bar{u} & \text { in } & \Omega \\
\int_{0}^{1} q d \tau=0 & \text { on } & \partial \Omega
\end{array}\right.
$$


again by Poincaré's inequality,

$$
\left\|\int_{0}^{1} q d \tau\right\|_{H_{0}^{1}(\Omega)} \leq \frac{C}{T}
$$

Therefore, from (5.10), (5.11) and (5.13), by the Cauchy-Schwartz inequality, we obtain (5.12). In particular,

$$
\lim _{T_{n} \rightarrow \infty} M_{T_{n}}(x)=M_{\infty}(x), \quad \text { a.e. } x \in \Omega .
$$

Step 3: conclusion. We first note that our assumption on $f$ implies that the set

$$
\omega_{0}=\{x \in \Omega: \nabla \bar{u}(x)=0\}
$$

has zero Lebesgue measure. Indeed, this is a consequence of the weak form of (5.8).

Thus, for almost every $x \in \Omega$ we have $\nabla \bar{u}(x) \neq 0$. Since the matrix $M_{\infty}(x)$ has rank 1 and is non-negative, by Lemma 5.1, there exists $T_{n_{0}}=T_{n_{0}}(x)$ such that for $T_{n} \geq T_{n_{0}}$ any solution of

$$
\max _{K^{0} \in G_{\theta_{T_{n}}}(x)} K^{0}: M_{T_{n}}(x)
$$

is a laminate of rank less than or equal to $N-1$. In particular, $K_{T_{n}}^{\star}(x)$ also is. As shown in the proof of Lemma 5.1, in the two-dimensional case it is in fact a first-order laminate. For $N \geq 3$, if for some index $0 \leq i \leq N-1$ the corresponding eigenvalue $\lambda_{n}^{i}$ of $M_{T_{n}}(x)$ is different from zero, then again by Lemma $5.1,(5.15)$ has only a solution which corresponds to a first-order laminate. However, if $\lambda_{n}^{i}=0$ for all $0 \leq i \leq N-1$, then we can only ensure that $K_{T_{n}}^{\star}(x)$ is a laminate of rank less than or equal to $N-1$.

6. Numerical experiments. In this section, we present a numerical approximation of $\left(R P_{T}\right)$ and then study for $N=2$ the behavior of the solution $\left(\theta_{T}, K_{T}^{\star}\right)$ with respect to $T$ in three specific examples.

6.1. Numerical resolution of $\left(R P_{T}\right)$. In Section 4 we proposed an algorithm, based on the optimality conditions. Actually, a tricky part of this so-called optimality criteria algorithm is to find a root $\theta$ of the equation $Q_{T}(x)=0$ where $Q_{T}$ is defined by (4.8). In order to avoid this difficulty, as well as for stability reasons, we modify this algorithm so that only $K_{T}^{\star}$ is updated thanks to the optimality condition (4.11), while $\theta$ is updated by a descent gradient method. For $N=2$, we refer to [11] for a pure gradient method based on a different parametrization of the optimal tensor $K_{T}^{\star}$. Relation (4.2) gives the descent direction for $\theta$ :

$$
\delta \theta=\frac{1}{T} \int_{0}^{T}\left[K_{T, \theta}^{\star} \nabla u \cdot(\nabla u+2 \nabla p)-2\left(\beta_{2}-\beta_{1}\right) u^{\prime} p\right] d t+l \quad \text { in } \Omega,
$$

where $K_{T, \theta}^{\star}$ denotes the first derivative of $K_{T}^{\star}$ with respect to $\theta$. Consequently, for any function $\eta \in L^{\infty}\left(\Omega, \mathbb{R}^{+}\right)$with $\|\eta\|_{L^{\infty}(\Omega)}$ small enough, we have $\bar{J}_{T}^{\star}(\theta+\eta \delta \theta) \leq \bar{J}_{T}^{\star}(\theta)$. The multiplier $l$ is determined writing that (for any $\left.\eta \in L^{\infty}\left(\Omega, \mathbb{R}^{+}\right)\right)\|\theta+\eta \delta \theta\|_{L^{1}(\Omega)}=$ $L|\Omega|$, which leads to

$$
l=\frac{\int_{\Omega} \theta d x-L|\Omega|+T^{-1} \int_{\Omega} \eta \int_{0}^{T}\left[K_{T, \theta}^{\star} \nabla u \cdot(\nabla u+2 \nabla p)-2\left(\beta_{2}-\beta_{1}\right) u^{\prime} p\right] d t d x}{\int_{\Omega} \eta d x} .
$$


At last, the function $\eta$ is chosen so that $\theta+\eta \delta \theta \in[0,1]$ for all $x \in \Omega$. A simple and efficient choice consists in taking $\eta=\epsilon \theta(1-\theta)$ with $\epsilon$ positive and small enough (we refer to [10] for more details).

In the sequel we denote by $\left(v_{1}, v_{2}, \cdots, v_{N}\right)$ the eigenvalues of $K_{T}^{\star}$ and $P=$ $\left(e_{1}, e_{2}, \cdots, e_{N}\right)$ the matrix of the corresponding eigenvectors so that $K_{T}^{\star}=P \Lambda_{T} P^{t}$ with $\Lambda_{T}=\operatorname{diag}\left(v_{1}, v_{2}, \cdots, v_{N}\right)$ and $P^{t}$ the transpose matrix of $P$.

Our algorithm to solve the relaxed problem $\left(R P_{T}\right)$ is as follows: given $T>0$, $L \in(0,1), \Omega \subset \mathbb{R}^{2}, u_{0}, f \in L^{2}(\Omega)$ and a small convergence threshold $0<\varepsilon<<1$,

- Initialization: take $\theta_{T}^{0}=L, \Lambda_{T}^{0} \in \partial G_{\theta}, P^{0}$ the identity matrix, so that $K_{T}^{\star, 0}=P^{0} \Lambda_{T}^{0}\left(P^{0}\right)^{t}$.

- For $n \geq 1$, iteration until convergence, which is detected when

$$
\left|\bar{J}_{T}^{\star}\left(\theta_{T}^{n-1}, K^{\star, n-1}\right)-\bar{J}_{T}^{\star}\left(\theta_{T}^{n}, K^{\star, n}\right)\right| \leq \varepsilon\left|\bar{J}_{T}^{\star}\left(\theta_{T}^{0}, K^{\star, 0}\right)\right| .
$$

1. Compute the state $u^{n}$ and the adjoint state $p^{n}$ by solving (2.3) and (4.3) respectively with the previous design parameters $\theta_{T}^{n-1}$ and $K_{T}^{\star, n-1}$.

2. Compute the descent direction $\delta \theta\left(u^{n}, p^{n}\right)$ given by (6.1) and the multiplier $l^{n}$ given by (6.2). The first derivative of $K_{T}^{\star, n-1}$ with respect to $\theta$ is given by $K_{T, \theta}^{\star, n-1}=P \Lambda_{T, \theta}^{n-1} P^{t}$ where the diagonal matrix $\Lambda_{T, \theta}^{n-1}$ is obtained by differentiating the eigenvalues $v_{i}, i=1, \ldots, N$ with respect to $\theta$ (given explicitly, for $N=2$, by the formula (4.16)). Then, update the density in $\Omega$ :

$$
\theta_{T}^{n}:=\theta_{T}^{n-1}+\eta \delta \theta\left(u^{n}, p^{n}\right)
$$

3. Compute the matrix $M_{T}^{n}(x)$ given by formula (4.5) which depends on $\left(u^{n}, p^{n}\right)$, its eigenvalues $\mu_{1}^{n}, \mu_{2}^{n}, \cdots, \mu_{N}^{n}$ and corresponding eigenvectors $e_{1}^{n}, e_{2}^{n}, \cdots, e_{N}^{n}$, and set $P^{n}=\left(e_{1}^{n}, e_{2}^{n}, \cdots, e_{N}^{n}\right)$.

4. Solve the linear optimization problem $\max _{K^{0} \in G_{\theta_{T}^{n}}} K^{0}: M_{T}^{n}$ (using, for $N=2$, the formula (4.16)) yielding the maximizer $\Lambda_{T}^{n} \in \partial G_{\theta_{T}^{n}}$ and $K_{T}^{\star, n}=P^{n} \Lambda_{T}^{n}\left(P^{n}\right)^{t}$.

Since the optimality condition is used to update the variable $K_{T}^{\star, n}$, we highlight that there is a priori no guarantee that this algorithm produces a minimizing sequence $\left(\theta_{T}^{n}, K_{T}^{\star, n}\right)$ for the functional $J$. In practice, we will observe however such a property.

Notice that the expression for $K_{T}^{\star, n}$ in Step 4 saturates Hashin-Shtrikman bounds and therefore we may compute the derivative of $K_{T}^{\star, n}$ with respect to $\theta$. The partial differential equations (2.3) and (4.3) are approximated with a $Q_{1}$ finite element method for the spatial discretization and with an implicit Euler scheme for the time discretization. In all our experiments, we take simply $\Omega=(0,1) \times(0,1)$ and use a uniform quadrangular mesh. The parameters for the meshes are $h=1 / 100$ and $d t=h / 2$ for the spatial and time discretization respectively.

6.2. Example 1: Uniform heat source $f$. A well-known and addressed example in the elliptic situation corresponds to a uniform load (see for instance [4]): we therefore take $f \equiv 1$ in $\Omega=(0,1)^{2}$. We put $u_{0}=0, \beta_{1}=\beta_{2}=1, k_{1}=0.07, k_{2}=2 k_{1}$, and take a volume fraction for the material $\left(\beta_{1}, k_{1}\right)$ equal to $50 \%$ corresponding to $L=0.5$ in (1.2). Table 6.1 reports the value of the optimal cost $J_{T}^{\star}\left(\theta_{T}, K_{T}^{\star}\right)$ for increasing values of $T$. The column $T=\infty$ corresponds to the value of $J_{\infty}^{\star}\left(\theta_{\infty}, K_{\infty}^{\star}\right)$ defined in Theorem 2.2. As expected, the cost converges exponentially towards $J_{\infty}^{\star}\left(\theta_{\infty}, K_{\infty}^{\star}\right)$. Figure 6.1 depicts the iso-values in $\Omega$ of the corresponding density $\theta_{T}$. In particular, 
we check that in the elliptic case - corresponding to the problem $\left(R P_{\infty}\right)$-, we obtain the well-known cross geometry (see Figure 6.1 bottom right). We observe that the composite zone $\left\{x \in \Omega, 0<\theta_{T}(x)<1\right\}$ is rather small. For $T=0.5,1,1.5,2,4$, the eigenvalues $\mu_{1}^{T}, \mu_{2}^{T}$ of the matrix $M_{T}$ satisfy $\mu_{1}^{T} \mu_{2}^{T} \leq 0$ at every node of the mesh. Consequently, at these nodes, the optimal micro-structure corresponds to a rank-one laminate. Figure 6.3 depicts the direction of lamination given by the first eigenvector of the optimal matrix $M_{T}$ for $T=2$ and $T=\infty$, of interest only in $\{x \in \Omega, 0<\theta(x)<1\}$. We observe that for $T$ large, the optimal direction of lamination is close to the direction of lamination associated with the elliptic case. The knowledge of the optimal density and of the lamination allows to construct a minimizing sequence of classical designs (see [14]). Figure 6.2-left depicts for $T=4$ the evolution of the cost $J_{T}^{\star}\left(\theta_{T}^{n}, K_{T}^{\star, n}\right)$, and Figure 6.2-right shows the evolution of the function $t \rightarrow 1 / 2 \int_{\Omega} K_{T}^{\star} \nabla u(t) \cdot \nabla u(t) d x$ for $0 \leq t \leq 4$. These results are obtained with the initialization $\left(v_{1}^{0}, v_{2}^{0}\right)=\left(\lambda_{\theta}^{-}, \lambda_{\theta}^{+}\right)$and seems to be independent on the initialization: for instance, we obtain the same result $\left(K_{T}^{\star}, \theta_{T}\right)$ if we consider $\left(v_{1}^{0}, v_{2}^{0}\right) \in \partial G_{\theta}$, with $v_{1}^{0}=v_{2}^{0}$.

TABLE 6.1

(Example 1) - Value of the optimal cost $J_{T}^{\star}\left(\theta_{T}, K_{T}^{\star}\right)$ vs. T.

\begin{tabular}{c|c|c|c|c}
$T=0.5$ & $T=1$ & $T=2$ & $T=4$ & $" T=\infty "$ \\
\hline $6.17 \times 10^{-2}$ & $1.26 \times 10^{-1}$ & $1.99 \times 10^{-1}$ & $2.49 \times 10^{-1}$ & $2.93 \times 10^{-1}$
\end{tabular}

6.3. Example 2 : Non-uniform heat source $f$. We now consider a nonuniform source term $f$ with both positive and negative values

$$
f(x)=\mathcal{X}_{(0.05,0.15) \times(0.1,0.9)}(x)-\mathcal{X}_{(0.85,0.95) \times(0.1,0.9)}(x)
$$

with a support arranged as two vertical strips, positive on the left, negative on the right. From a physical point of view we expect to have the good conductor mainly placed between those two strips. However, if the proportion of the good conductor phase is not large enough, it should mix itself with the other phase as a rank-one laminate with horizontal layers. This example has thus been cooked up to get an optimal design with large zone of composites. We take $k_{1}=0.035$ and $k_{2}=2 k_{1}$ while the rest of numerical values are unchanged. Figure 6.4 depicts the iso-values of the optimal density $\theta_{T}$ as well as the direction of lamination, for $T=0.25,0.5,1,2,4$ and $T=\infty$. We observe a transition in the direction of lamination : for $T$ small, the laminates are oriented along $\left(O x_{1}\right)$, while for $T$ large, the laminates are oriented along $\left(O x_{2}\right)$. Corresponding numerical values of the cost are given in Table 6.2. This example produces a larger zone of composite than the first one, especially for extreme values of $T$. However, we observe once again numerically that the corresponding optimal tensor $K_{T}^{\star}$ is recovered by a laminate of rank one.

TABLE 6.2

(Example 2) - Value of the optimal cost $J^{\star}\left(\theta_{T}, K_{T}^{\star}\right)$ vs. T.

\begin{tabular}{c|c|c|c|c}
$T=0.25$ & $T=0.5$ & $T=2$ & $T=4$ & $" T=\infty "$ \\
\hline $5.238 \times 10^{-3}$ & $7.909 \times 10^{-3}$ & $1.30 \times 10^{-2}$ & $1.412 \times 10^{-2}$ & $1.540 \times 10^{-2}$
\end{tabular}



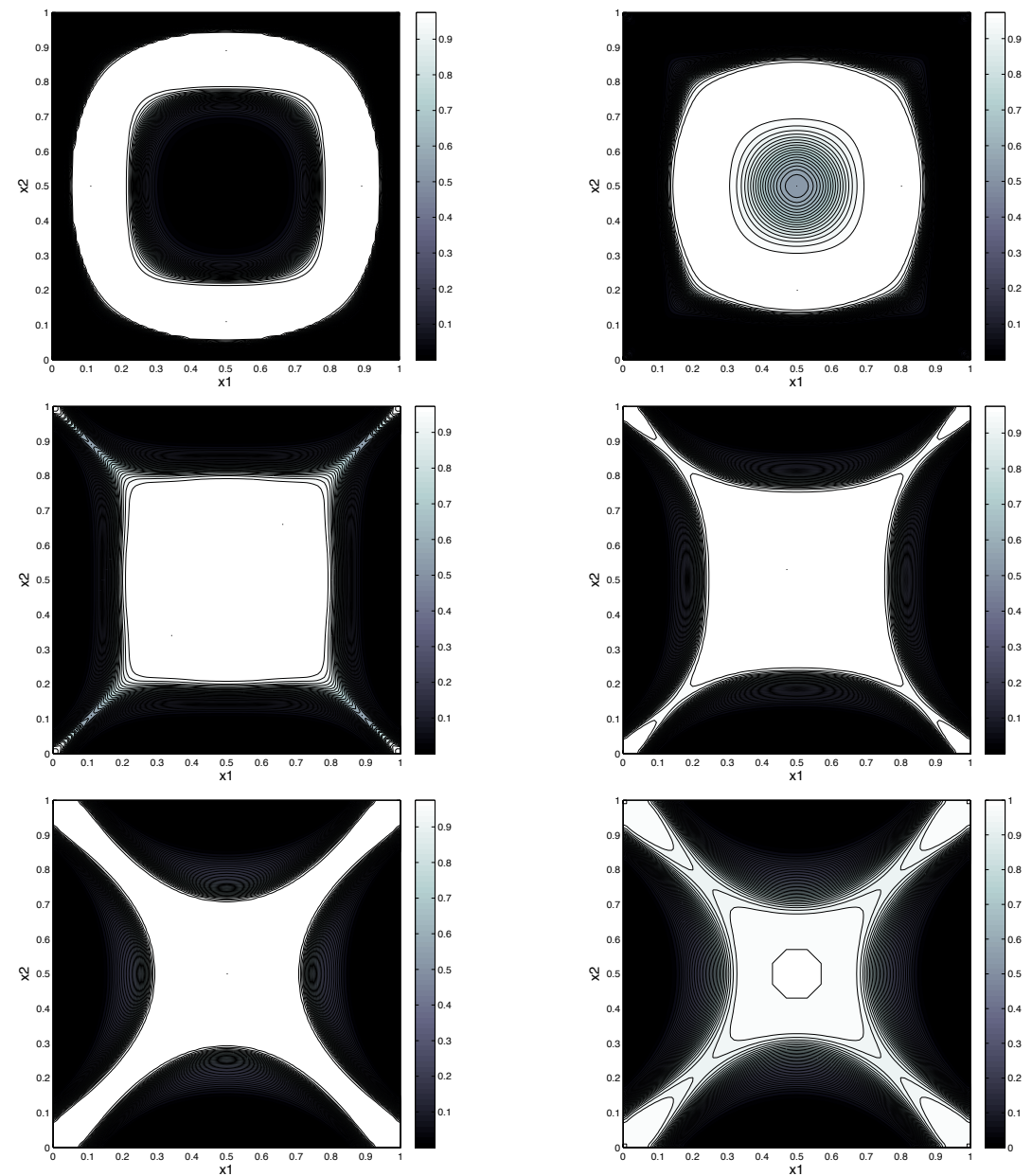

FIG. 6.1. (Example 1) - Iso-values of $\theta_{T}$ in $\Omega$ : the white color corresponds to the smallest conductivity. From left to right and top to down, $T=0.5, T=1, T=1.5, T=2, T=4$ and the limit elliptic case " $T=\infty "$.
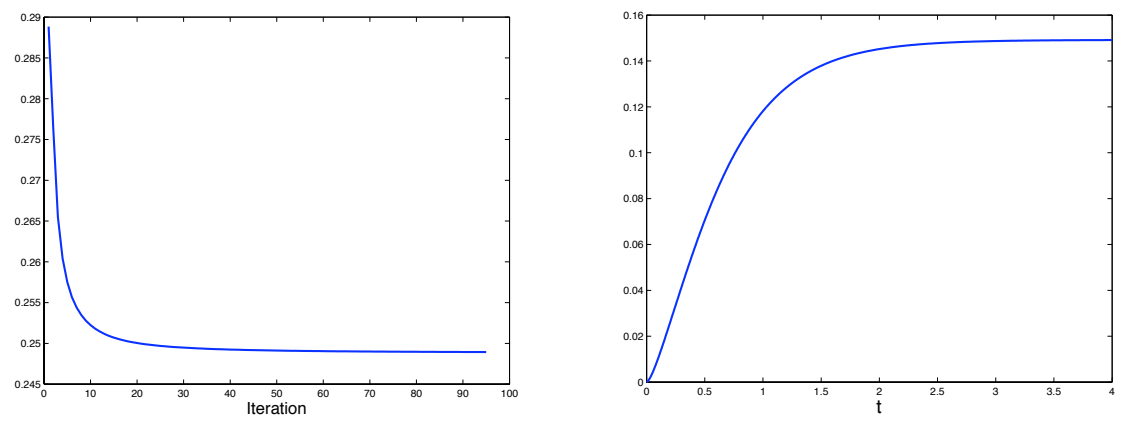

FIG. 6.2. (Example 1) - For $T=4$, convergence history $n \rightarrow J_{T}^{\star}\left(\theta_{T}^{n}, K_{T}^{\star, n}\right)$ (Left) and evolution of the stored energy $t \rightarrow 1 / 2 \int_{\Omega} K_{T}^{\star} \nabla u(t) \cdot \nabla u(t) d x$ (Right). 

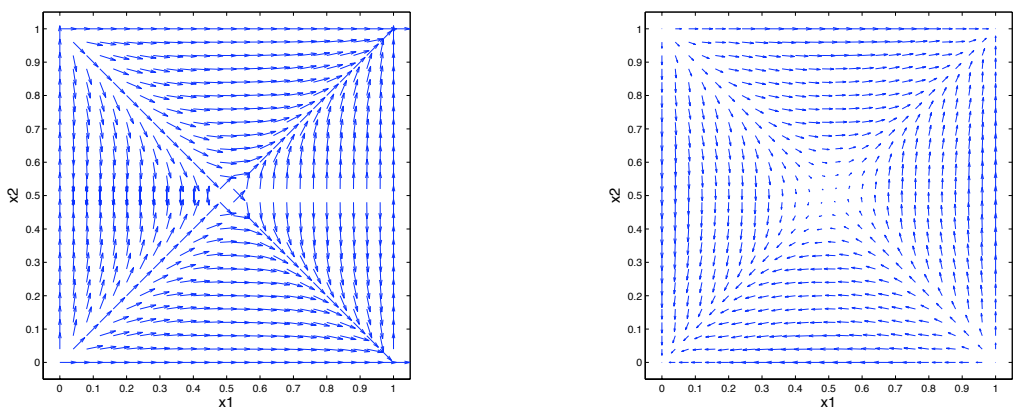

FIG. 6.3. (Example 1) - Direction of lamination for $T=2$ (Left) and $T=\infty$ (Right).
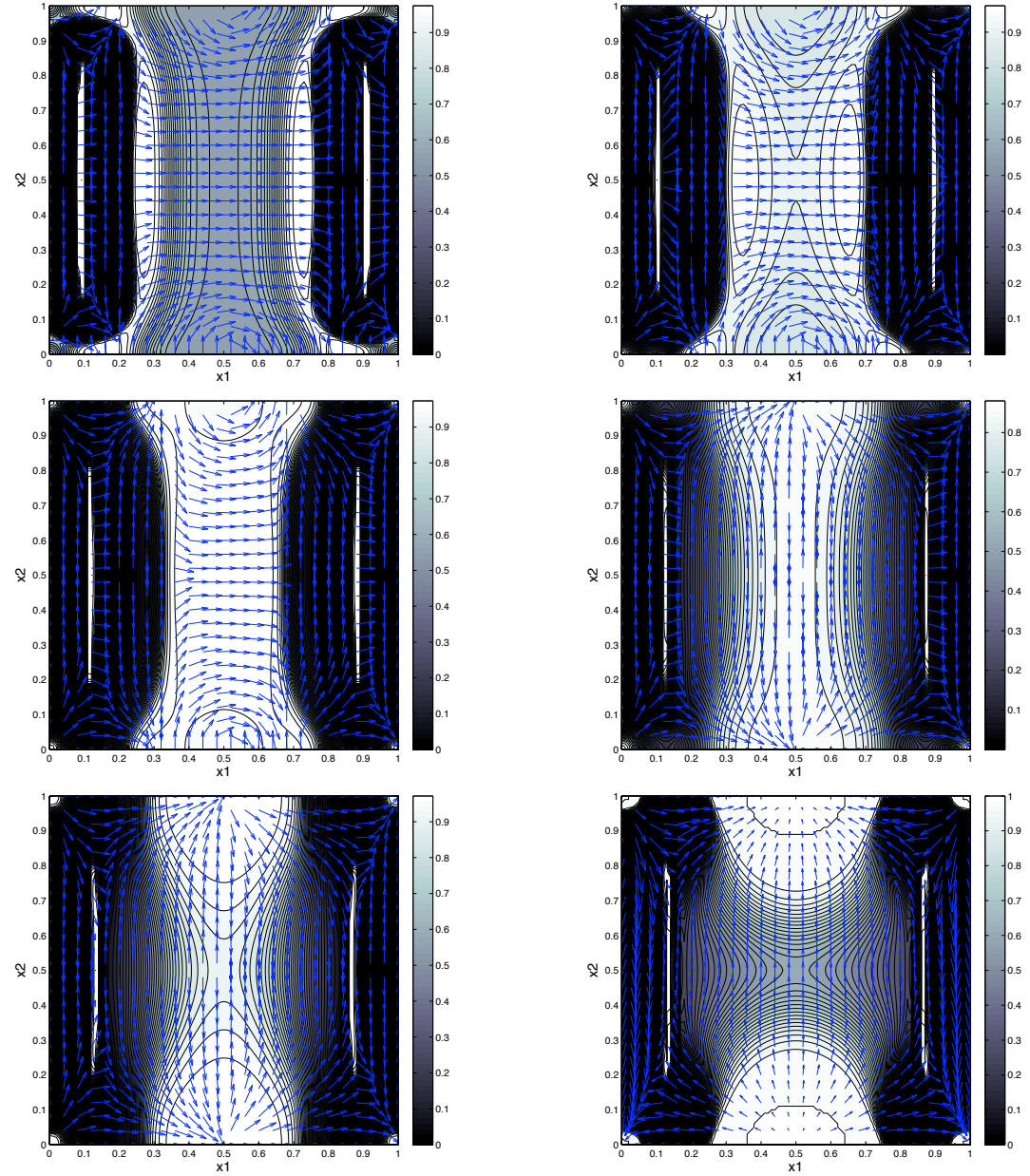

FIG. 6.4. (Example 2) - Iso-values of $\theta_{T}$ and direction of lamination in $\Omega$. From left to right and top to bottom, $T=0.25, T=0.5, T=1, T=2, T=4$ and the limit elliptic case " $T=\infty "$.

6.4. Example 3: Interplay between $f$ and $u_{0}$ - Second order laminates. In this third example, we keep unchanged the data from the previous example, except 
that we now take a non-zero initial condition

$$
u_{0}(x)=\frac{1}{4} \mathcal{X}_{(0.2,0.8) \times(0.1,0.2)}(x)-\frac{1}{4} \mathcal{X}_{(0.2,0.8) \times(0.8,0.9)}(x)
$$

with a support made of two horizontal strips, positive at the bottom, negative at the top. For small enough time $T$, the effect of the source term is negligible and the two phases should arrange themselves between these two horizontal strips as a rank-one laminate with vertical layers. Therefore, as the final time $T$ increases, we expect a transition from vertical layers to horizontal ones and possibly the occurrence of some rank-two laminates. Our numerical experiments are in agreement with this prediction. Figure 6.5 depicts the iso-values of the optimal density for $T=0.125,0.25,0.5$ and $T=1$. The subset of $\Omega$ corresponding to second order laminates is plotted on Figure 6.6. The subset of rank-two laminates is obtained from the optimality condition (4.16) evaluated at each node of the spatial mesh. Finally, Figure 6.7 depicts the first eigenvector of $M_{T}$ which gives the orientation of the optimal microstructure.
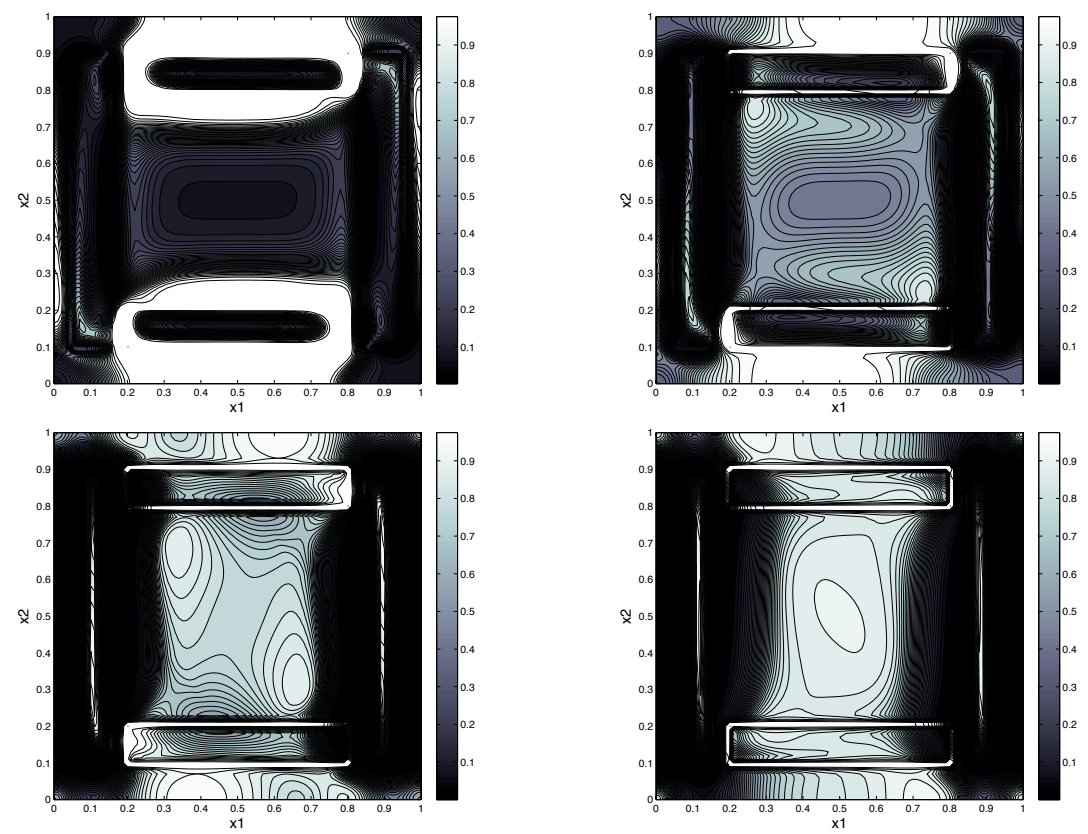

FIG. 6.5. (Example 3) - Iso-values of $\theta_{T}$ in $\Omega$. From left to right and top to bottom, $T=$ $0.125,0.25,0.5$ and $T=1$.

TABLE 6.3

(Example 3) - Value of the optimal cost $J^{\star}\left(\theta_{T}, K_{T}^{\star}\right)$ vs. T.

\begin{tabular}{c|c|c|c|c}
$T=0.125$ & $T=0.5$ & $T=1$ & $T=4$ & $" T=\infty "$ \\
\hline $2.98 \times 10^{-2}$ & $1.69 \times 10^{-2}$ & $1.60 \times 10^{-2}$ & $1.58 \times 10^{-2}$ & $1.54 \times 10^{-2}$
\end{tabular}

Acknowledgments- This work was partially done while the second author was visiting the "Centre de Mathématiques Appliquées" of the Polytechnic School (Palaiseau - France). He wishes to thank their members for the kind hospitality. 

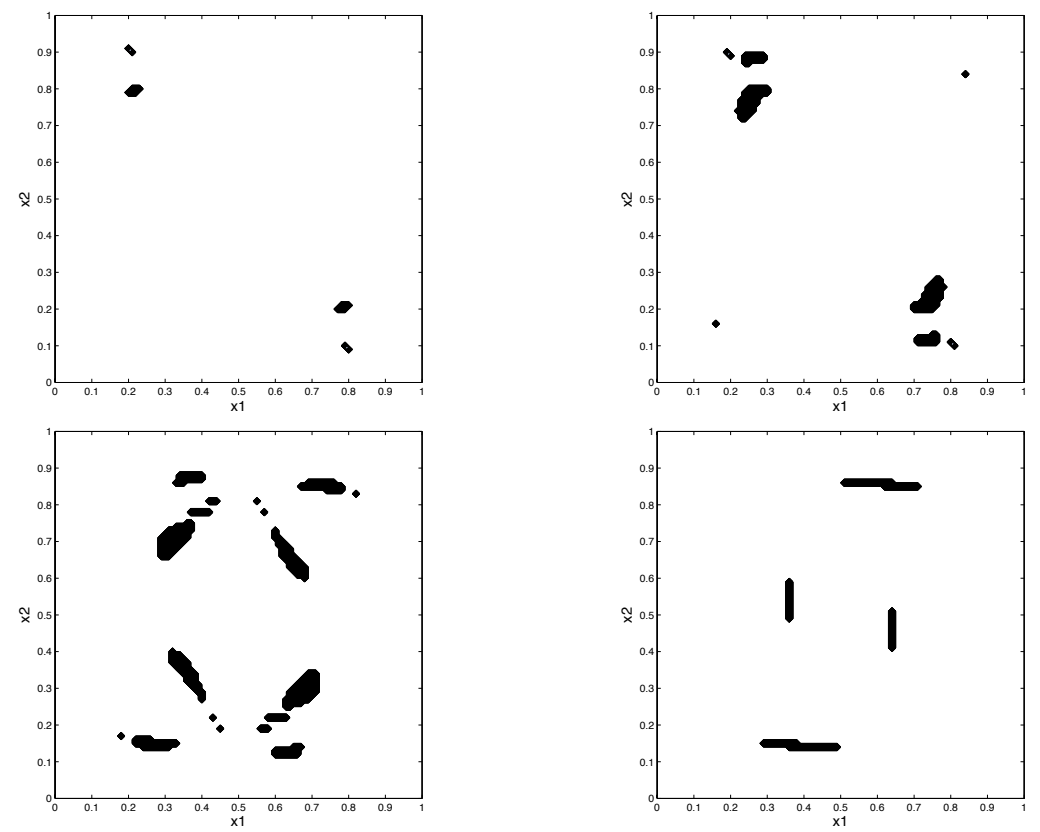

FIG. 6.6. (Example 3) - Second order laminate zone in $\Omega$ for $T=0.125,0.25,0.5$ and $T=1$ (from left to right and top to bottom).
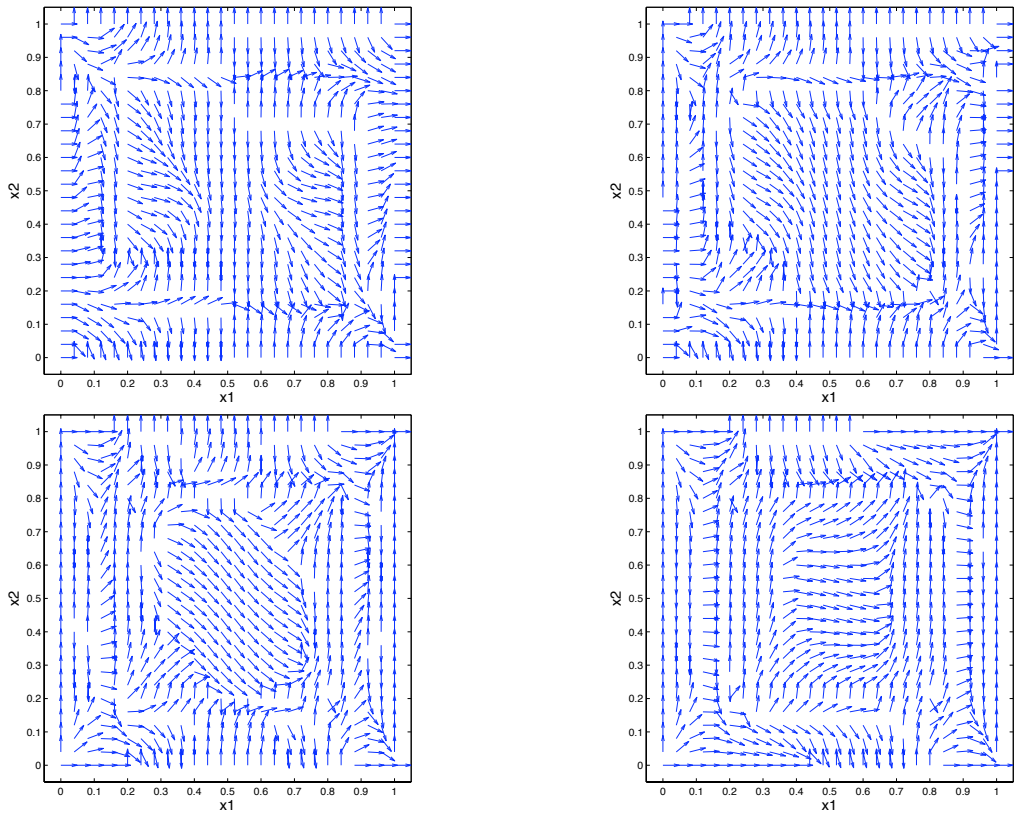

FIG. 6.7. (Example 3) - First eigenvector of the matrix $M_{T}$ for $T=0.125,0.25,0.5$ and $T=1$.

\section{REFERENCES}

[1] G. Allaire, Shape Optimization by the Homogenization Method, Applied Mathematics Sci- 
ences 146, Springer, 2002.

[2] N. Antonić, AND M. VRdolJak, Gradient methods for multiple state optimal design problems, Ann. Univ. Ferrara 53 (2007) pp. 177-187.

[3] A. Cherkaev, Variational methods for structural optimization, Springer-Verlag, New-York, 2000.

[4] R. GLOwINSKI, Numerical simulation for some problems originating from continuum mechan$i c s$, in Trends in applications of pure mathematics to mechanics, Symp. Palaiseau/France 1983, Lecture Notes in Physics 195, (1984) pp. 96-145.

[5] J. S. JENSEn, Space-time topology optimization for one-dimensional wave propagation, Comput. Methods Appl. Mech. Engrg. 198 (2009) pp. 705-715.

[6] R.V. Kohn, and G. Strang, Optimal design and relaxation of variational problems, Comm. Pure Appl. Math. 39 (1986) pp. 113-182.

[7] J. L. Lions, And E. MAGenes, Non-Homogeneous boundary values problems and its applications, Vol. 1, Springer-Verlag, 1972.

[8] K. A. LuRIE, Control in the coefficients of linear hyperbolic equations via spatio-temporal components. Homogenization, pp. 285-315, Ser. Adv. Math. Appl. Sci., 50, World Sci. Publ., River Edge, NJ, 1999.

[9] K. A. LuRIE, Some new advances in the theory of dynamic materials, Journal of Elasticity 72 (2003), pp. 229-239.

[10] F. Maestre, A. MÜnCh, and P. Pedregal, A spatio-temporal design problem for a damped wave equation, SIAM J. Applied Mathematics 68(1), (2007) pp. 109-132.

[11] A. Münch, P. Pedregal, and F. Periago, Relaxation of an optimal design problem for the heat equation, J. Math. Pures Appl. 89 (2008) pp. 225-247.

[12] F. Murat, Contre-exemples pour divers problèmes où le contrôle intervient dans les coeffcients, Ann. Mat. Pura Appl. 112 (1977) pp. 49-68.

[13] F. Murat, and L. Tartar, Calcul des Variations et Homogénéisation, Les Méthodes d'Homogénéisation Théories et Applications en Physique, Coll. Dir. Etudes et Recherches EDF, 57, Eyrolles, Paris, (1985) pp. 319-369. "English translation in Topics in the mathematical modelling of composite materials, A. Cherkaev and R. Khon editors, Progress in Nonlinear Differential Equations and their Applications, 31, Birkhäuser, Boston (1997)."

[14] O. Pantz, And K. Trabelsi, A post-treatment of the homogenization method for shape optimization, SIAM J. Control Optim. 47 (2008) pp. 1380-1398.

[15] P. Pedregal, Vector variational problems and applications to optimal design, ESAIM-COCV, 11(3), (2005) pp. 357-381.

[16] P. Pedregal, Optimal design in two-dimensional conductivity for a general cost depending on the field, Arch. Rational Mech. Anal. 182 (2006), pp. 367-385.

[17] U. RAITUms, The extension of extremal problems connected with a linear elliptic equation, Soviet Math. Lecture notes in Mathematics, 19 (1978) pp. 1342-1345.

[18] L. TARTAR, An introduction to the Homogenization method in optimal design, Lecture notes in Mathematics, 1740, Eds. A. Cellina, A. Ornelas, (1998) pp. 47-156.

[19] S. Turteltaub, Optimal material properties for transient problems, Struct. Multidiscipl. Optim. 22, (2001) pp. 157-166.

[20] S. Turteltaub, Functionally graded materials for prescribed field evolution, Compt. Methods Appl. Mech. Engrg. 192, (2002) pp. 2283-2296.

[21] M. VRDOLJAK, Necessary conditions for optimal design in hyperbolic problems, Applied mathematics and scientific computing, Tambaca, Josip et al., editors. Zagreb, 2007.

[22] M. VRDOLJAK, Multiple state optimal design problems and Hashin-Shtikman bounds, Int. J. Pure Appl. Math.,42 (2008) pp. 319-324. 